\title{
A New Three-Parameter Weibull Inverse Rayleigh Distribution: Theoretical Development and Applications
}

\author{
Adeyinka Solomon Ogunsanya ${ }^{1, *}$, Waheed Babatunde Yahya ${ }^{1}$, Taiwo Mobolaji Adegoke ${ }^{1}$, \\ Christiana Iluno ${ }^{2}$, Oluwaseun R. Aderele ${ }^{2}$, Matthew Iwada Ekum ${ }^{2}$ \\ ${ }^{1}$ Department of Statistics, University of Ilorin, Ilorin, Nigeria \\ ${ }^{2}$ Department of Mathematics and Statistics, Lagos State Polytechnic, Ikorodu, Lagos, Nigeria
}

Received December 11, 2020; Revised February 4, 2021; Accepted March 12, 2021

\begin{abstract}
Cite This Paper in the following Citation Styles
(a): [1] Adeyinka Solomon Ogunsanya, Waheed Babatunde Yahya, Taiwo Mobolaji Adegoke, Christiana Iluno, Oluwaseun R. Aderele, Matthew Iwada Ekum , "A New Three-Parameter Weibull Inverse Rayleigh Distribution: Theoretical Development and Applications," Mathematics and Statistics, Vol. 9, No. 3, pp. 249 - 272, 2021. DOI: 10.13189/ms.2021.090306.
\end{abstract}

(b): Adeyinka Solomon Ogunsanya, Waheed Babatunde Yahya, Taiwo Mobolaji Adegoke, Christiana Iluno, Oluwaseun R. Aderele, Matthew Iwada Ekum (2021). A New Three-Parameter Weibull Inverse Rayleigh Distribution: Theoretical Development and Applications. Mathematics and Statistics, 9(3), 249 - 272. DOI: 10.13189/ms.2021.090306.

Copyright $\bigcirc 2021$ by authors, all rights reserved. Authors agree that this article remains permanently open access under the terms of the Creative Commons Attribution License 4.0 International License

\begin{abstract}
In this work, a three-parameter Weibull Inverse Rayleigh (WIR) distribution is proposed. The new WIR distribution is an extension of a one-parameter Inverse Rayleigh distribution that incorporated a transformation of the Weibull distribution and Log-logistic as quantile function. The statistical properties such as quantile function, order statistic, monotone likelihood ratio property, hazard, reverse hazard functions, moments, skewness, kurtosis, and linear representation of the new proposed distribution were studied theoretically. The maximum likelihood estimators cannot be derived in an explicit form. So we employed the iterative procedure called Newton Raphson method to obtain the maximum likelihood estimators. The Bayes estimators for the scale and shape parameters for the WIR distribution under squared error, Linex, and Entropy loss functions are provided. The Bayes estimators cannot be obtained explicitly. Hence we adopted a numerical approximation method known as Lindley's approximation in other to obtain the Bayes estimators. Simulation procedures were adopted to see the effectiveness of different estimators. The applications of the new WIR distribution were demonstrated on three real-life data sets. Further results showed that the new WIR distribution performed credibly well when compared with five of the related existing skewed distributions. It was observed that the Bayesian estimates derived performs better than the classical method.
\end{abstract}

Keywords Weibull, Weibull Inverse Rayleigh Distribution, Monotone Likelihood Ratio, Order Statistic, Weibull Distribution

\section{Introduction}

In probability theory and statistics, a generalized lifetime model suitable for fitting survival and engineering data is often of interest in the survival and reliability analysis [1]. Convoluted distributions derived from the $\mathrm{T}-\mathrm{R}\{\mathrm{Y}\}$ framework by [2] and [3] have the probability density function (PDF) that are weighted hazard function of a baseline distribution, which can provide insight into characteristics of failure times and hazard functions that may not be available with classical models.

Many authors have derived hybrid or convoluted distributions that are members of this family, especially the ones that were derived using the standard quantile function of the log-logistic distribution such as Weibull-Uniform $\{$ Log-logistic $\}$ by [2]; Exponential-Normal $\{$ Log-logistic $\}[3]$;

Lomax-Cauchy $\{$ Log-logistic $\}[4] ; \quad$ Weibull-Gamma \{Log-logistic\} [5]; Weibull-Exponential\{Log-logistic\}, Gamma-Exponential\{Log-logistic $\} \quad$ and Normal-Exponential \{ Log-logistic $\} \quad\left[\begin{array}{lll}6 & 6\end{array}\right]$; Weibull-Normal \{ Log-logistic \} [7]; and [8]which 
exploited the statistical properties of Odd Lomax-Exponential\{Log-logistic $\}$ distribution. All distributions are members of the T-R $\{\log$-logistic $\}$ family that have a probability density function with three components vis-a-vis the hazard function, survival function, and the odd-ratio. These three components are very important in the survival analysis. The distributions of interest in this research are the Weibull and Inverse Rayleigh distributions.

Several researchers have exploited the importance of Inverse Rayleigh distribution in social, medical, and engineering fields. The extension and generalization of Inverse Rayleigh distribution were studied by some authors. [9] studied some properties of Exponentiated Transmuted Generalized Rayleigh distribution, [10] proposed Half- Logistics Inverse Rayleigh distribution, [11] studied type II Topp-Leone inverse Rayleigh distribution, [12] Transmuted inverse Rayleigh distribution (TIR), [13] fitted the same data on odd Frechet inverse Rayleigh distribution (OFIR) and one parameter Inverse Rayleigh distribution. The possibility of using inverse Rayleigh distribution in this regard has not been accessed.

In this present study, a new member of the family of Weibull-G distribution defined by [14] through the $\mathrm{T}-\mathrm{R}\{\mathrm{Y}\}$ transformation was proposed. This new proposal is called the Weibull-Inverse Rayleigh \{Log-logistic $\}$ distribution having three parameters. Different functional properties of this new distribution are derived and their applications to survival and reliability analysis were presented using three real-life datasets, two of which were on corona-Virus 2019 (COVID-19), and the third one was on the material strength of carbon fiber.

The remaining parts of this article are arranged thus. In Section 2, the new three-parameters Weibull-inverse Rayleigh (WIR) distribution was proposed and its functional forms were presented. Some of its statistical properties were investigated in Section 3 while the procedures for parameter estimations were presented in Section 4. Applications of the new WIR distribution to three real-life data sets were provided in Section 5 while Section 6 presented the conclusion to the work.

\section{Theoretical Development}

In the $\mathrm{T}-\mathrm{R}\{\mathrm{Y}\}$ framework by [2], the random variable $T$ is a transformer that is used to transform the random variable $R$ into a new family of generalized distributions of $R$ using the quantile function of $Y$. If $Y$ follows the $\log$-logistic distribution, the standard quantile function of $Y$ as a function of the Cumulative Distribution Function (CDF) of $R$ gives the odd-ratio of $R$. Thus, the CDF of the generalized family of distributions as defined by [14] is given by

$$
F_{X}(x)=\int_{a}^{\left[\frac{G_{R}(x)}{1-G_{R}(x)}\right]} f_{T}(t) d t=F_{T}\left[\frac{G_{R}(x)}{1-G_{R}(x)}\right]
$$

and the corresponding probability density function (PDF) of $x, f(x)$ is derived by differentiating (1) with respect to $x$ to have

$$
f_{X}(x)=\frac{g_{R}(x)}{\left[1-G_{R}(x)\right]^{2}} f_{T}\left[\frac{G_{R}(x)}{1-G_{R}(x)}\right] .
$$

Alternatively, the PDF in (2) can be written as

$$
f_{X}(x)=\frac{h_{R}(x)}{S_{R}(x)} f_{T}\left[O_{R}(x)\right]
$$

where $h_{R}(x)$ is the hazard function of $R, S_{R}(x)$ is the survival function of $R$ and $O_{R}(x)$ is the odd-ratio of $R$. Let $T$ follows the Weibull distribution with CDF given by

$$
F_{T}(t)=1-\exp \left(-\alpha t^{\beta}\right)
$$

its corresponding PDF is given by

$$
f_{T}(t)=\alpha \beta t^{\beta-1} \exp \left(-\alpha t^{\beta}\right) ; \alpha, \beta>0 ; t \geq 0
$$

By using the CDF of [14], given in (1), we derived the Weibull-G family by putting (4) in (1) to have

$$
F_{X}(x)=1-\exp \left\{-\alpha\left[\frac{G_{R}(x)}{1-G_{R}(x)}\right]^{\beta}\right\}
$$

where $G_{R}(x)$ is the non-decreasing $\mathrm{CDF}$ of any known continuous probability distribution. The corresponding PDF to the Weibull-G family is derived by differentiating (5) with respect to $x$ to have

$$
f_{X}(x)=\frac{\alpha \beta t^{\beta-1} g_{R}(x)}{\left[1-G_{R}(x)\right]^{2}} \exp \left\{-\alpha\left[\frac{G_{R}(x)}{1-G_{R}(x)}\right]^{\beta}\right\} ; \alpha, \beta>0 ; t \geq 0
$$

\subsection{The New Three-Parameters Weibull-Inverse Rayleigh (WIR) Distribution}

Let $R$ be a random variable that follows the Inverse Rayleigh distribution with CDF given by

$$
G_{R}(r)=\exp \left(-(\delta / r)^{2}\right) .
$$

with the corresponding PDF given by

$$
g_{R}(r)=\frac{2 \delta^{2}}{r^{2}} \exp \left(-(\delta / r)^{2}\right) .
$$

By using the Weibull-G framework given in (5), we derived the CDF of Weibull Inverse Rayleigh (WIR) distribution by putting (7) in (5) to have

$$
F_{X}(x)=1-\exp \left\{-\alpha\left[\frac{\exp \left(-(\delta / x)^{2}\right)}{1-\exp \left(-(\delta / x)^{2}\right)}\right]^{\beta}\right\} .
$$

Solving (8) further to have

$$
F_{X}(x)=1-\exp \left\{-\alpha\left[\exp \left((\delta / x)^{2}\right)-1\right]^{-\beta}\right\}
$$

and the corresponding PDF is derived by differentiating (9) with respect to $x$ to have 


$$
\begin{gathered}
f_{X}(x)=\frac{2 \alpha \beta \delta^{2}}{x^{3}} \exp \left[-\beta(\delta / x)^{2}\right]\{1-\exp [-(\delta / \\
\left.\left.x)^{2}\right]\right\}^{-\beta-1} \times \exp \left\{-\alpha\left[\exp \left((\delta / x)^{2}\right)-1\right]^{-\beta}\right\} \\
\text { Density plot of WIRD }
\end{gathered}
$$

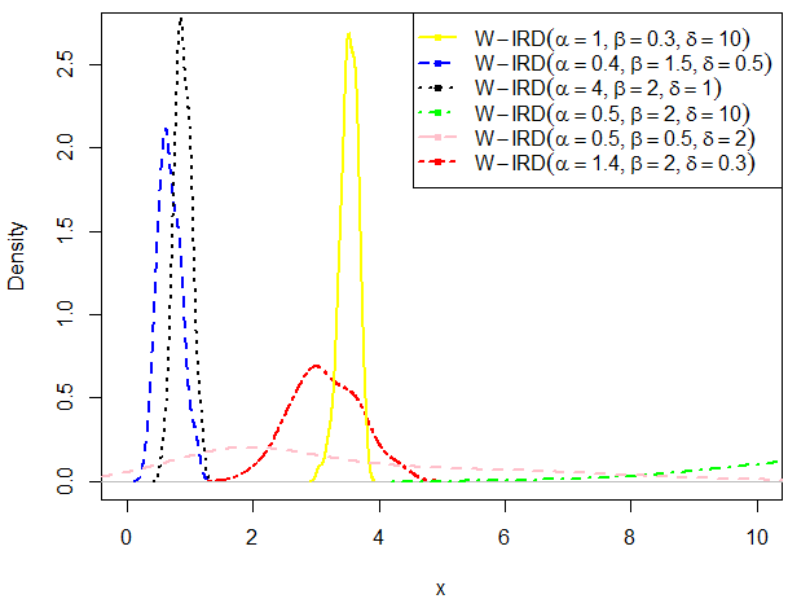

Figure 1. Density Plot of WIR Distribution

where $\beta$ is a shape parameter, $\alpha$ and $\delta$ are scale parameters. Thus, the CDF in (9) and the PDF in (10) are the CDF and PDF of the proposed three-parameters WIR distribution. The PDF of WIR distribution is demonstrated with different shape and scale parameters are shown in Figure 1.

\section{Properties of the New WIR Distribution}

The structural properties of the new WIR distribution are investigated in detail in this section. For ease, a random variable $X$ with $\mathrm{PDF} f_{X}(x)$ in (10) is said to follow the three-Parameters Weibull Inverse Rayleigh distribution and is denoted by $\mathrm{W}-\mathrm{IR}(\alpha, \beta, \delta)$.

\subsection{The Survival Function}

The survival function of the $\operatorname{WIR}(\alpha, \beta, \delta)$ is given by

$$
S_{X}(x)=\exp \left\{-\alpha\left[\exp \left((\delta / x)^{2}\right)-1\right]^{-\beta}\right\}
$$

The function in (11) is a function that gives the probability that an item of interest will survive beyond any given specified time $x \in X$, also known as the reliability function.

\subsection{The Hazard Function}

The hazard function of the $\operatorname{WIR}(\alpha, \beta, \delta)$ is given by

$h_{X}(x)=\frac{2 \alpha \beta \delta^{2}}{x^{3}} \exp \left[-\beta(\delta / x)^{2}\right]\left\{1-\exp \left[-(\delta / x)^{2}\right]\right\}^{-\beta-1}$

The function in (12) of random variables $X$ that follows WIR distribution is called the hazard rate of W-IR distribution, also known as the failure rate or force of mortality.

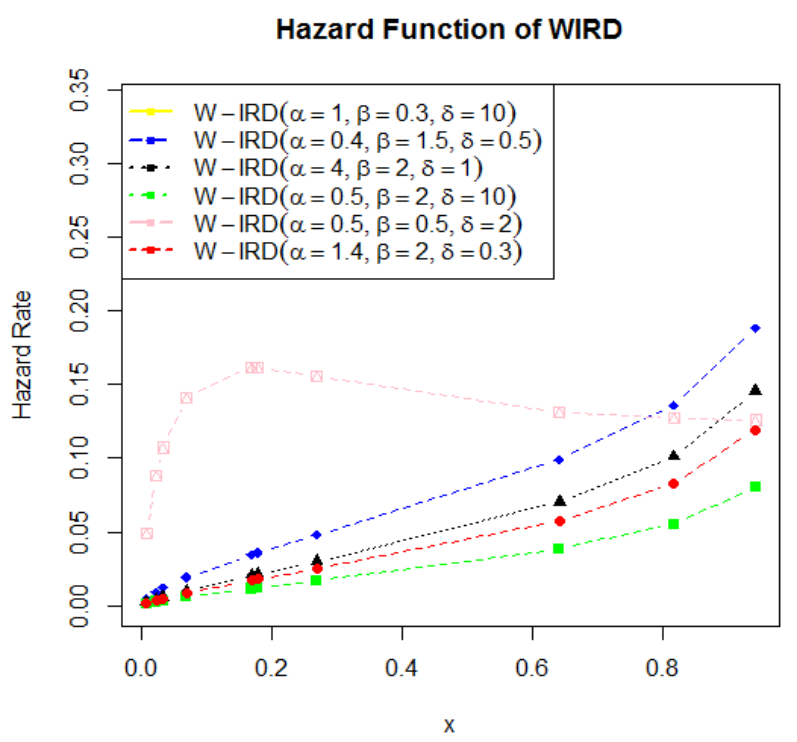

Figure 2. Hazard Function Plot of WIR Distribution

Different shapes of hazard function of WIR are displayed in Figure 2 with different values of scale and shape parameters.

\subsection{The Cumulative Hazard Function}

The cumulative hazard function, $H_{X}(x)$ of a continuous random variable $X$ that follows the WIR distribution is derived from this definition

$$
H_{X}(x)=-\log \left[S_{X}(x)\right]
$$

Put equation (11) into (13) to have

$$
H_{X}(x)=\alpha\left[\exp \left((\delta / x)^{2}\right)-1\right]^{-\beta}
$$

Equation (14) defines the probability of failure at time $x$ given survival until time $\forall x \in X$.

\subsection{The Reverse Hazard Function}

Given a random variable of $x$ that has a WIR distribution, the Reverse Hazard function of $x$ is given by

$$
\begin{gathered}
\tau_{X}=\frac{f(x)}{F(x)} \\
\tau_{X}= \\
\frac{\frac{2 \alpha \beta \delta^{2}}{x^{3}} \exp \left[-\beta(\delta / x)^{2}\right]\left\{1-\exp \left[-(\delta / x)^{2}\right]\right\}^{-\beta-1} \times \exp \left\{-\alpha\left[\exp \left((\delta / x)^{2}\right)-1\right]^{-\beta}\right\}}{1-\exp \left\{-\alpha\left[\exp \left((\delta / x)^{2}\right)-1\right]^{-\beta}\right\}}
\end{gathered}
$$

\subsection{The Median, Skewness, and Kurtosis}

Lemma 1: Let $T$ be a random variable that follows a Weibull distribution with parameters $\alpha$ and $\beta$, then the quantile function of $\operatorname{WIR}(\alpha, \beta, \delta)$ is given by 


$$
Q_{X}(p)=\frac{\delta}{\left\{\log \left(\frac{1+Q_{T}(p)}{Q_{T}(p)}\right)\right\}^{1 / 2}}
$$

where $Q_{T}(p)$ is the quantile function of Weibull distribution with parameters $\alpha$ and $\beta$.

\section{Proof.}

Using the concept of the T-R $\{\mathrm{Y}\}$ framework. If $X$ follows T-Inverse Rayleigh $\{\mathrm{Y}\}$ distribution, then it follows that

$$
X \stackrel{d}{=} \frac{\delta}{\left\{\log \left[\frac{1}{F_{Y}(T)}\right]\right\}^{1 / 2}} .
$$

This implies that

$$
Q_{X}(p)=\frac{\delta}{\left\{\log \left[\frac{1}{F_{Y}\left(Q_{T}(p)\right)}\right]\right\}^{1 / 2}}
$$

where $F_{Y}(x)$ is the CDF of a standard log-logistic distribution with all parameters equal to one, and it is given by

$$
F_{Y}(x)=\frac{x}{1+x}
$$

so that

$$
F_{Y}\left(Q_{T}(p)\right)=\frac{Q_{T}(p)}{1+Q_{T}(p)}
$$

Put equation (18) in (17) to have

$$
Q_{X}(p)=\frac{\delta}{\left\{\log \left(\frac{1+Q_{T}(p)}{Q_{T}(p)}\right)\right\}^{1 / 2}}
$$

Equation (19) completes the proof. The quantile function of $\operatorname{WIR}(\alpha, \beta, \delta)$ distribution in equation (19) can be computed from the Weibull quantile function by using $\mathrm{R}$ code $(T=\operatorname{rweibull}(p, \beta, \alpha))$, where $p$ is uniformly distributed on the closed interval 0 and 1 .
Theorem 1: Let $X$ be a random variable that follows a $\operatorname{WIR}(\alpha, \beta, \delta)$, its quantile function is given by

$$
Q_{X}(p)=\frac{\delta}{\left(\log \left\{1+\left[-\alpha^{-1} \log (1-p)\right]^{-1 / \beta}\right\}\right)^{1 / 2}}
$$

\section{Proof.}

From Lemma 1, it follows that $Q_{T}(p)$ is the quantile function of Weibull distribution with parameters $\alpha$ and $\beta$ given by

$$
Q_{T}(p)=[-\alpha \log (1-p)]^{1 / \beta}
$$

Substitute equation (20) into (19) to arrive at the quantile function of $\operatorname{WIR}(\alpha, \beta, \delta)$ and it is given by

$$
{ }_{x}(p)=\frac{\delta}{\left(\log \left\{1+\left[-\alpha^{-1} \log (1-p)\right]^{-1 / \beta}\right\}\right)^{1 / 2}}
$$

\subsubsection{Median}

The median and other measures of partition for the new WIR distribution can be derived from the quantile function in equation (21).

The median of $\operatorname{WIR}(\alpha, \beta, \delta)$ is given by

$$
M_{e}=Q_{X}(0.5)=\frac{\delta}{\left(\log \left\{1+\left[-\alpha^{-1} \log (1 / 2)\right]^{-1 / \beta}\right\}\right)^{1 / 2}}
$$

\subsubsection{The Skewness and Kurtosis}

The coefficient of skewness and kurtosis are mostly derived from the moment of a distribution. But we shall adopt measures of skewness and kurtosis based on quantile functions. The measure of skewness $S$ and kurtosis $K$ defined by [15] and [16] respectively are based on quantile functions and they are defined as

$$
S=\frac{Q(6 / 8)-2 Q(4 / 8)+Q(2 / 8)}{Q(6 / 8)-Q(2 / 8)}
$$

and

$$
K=\frac{Q(7 / 8)-Q(5 / 8)+Q(3 / 8)-Q(1 / 8)}{Q(6 / 8)-Q(2 / 8)}
$$


Theorem 2: The skewness and kurtosis of WIR distribution do not depend on parameter $\delta$.

Proof. Recall the quantile function of WIR distribution given in (21) as

$$
Q_{X}(p)=\frac{\delta}{\left(\log \left\{1+\left[-\alpha^{-1} \log (1-p)\right]^{-1 / \beta}\right\}\right)^{1 / 2}}
$$

Put (21) in (23) to have

$$
S=\frac{\frac{\delta}{\left(\log \left\{1+\left[-\alpha^{-1} \log (2 / 8)\right]^{-1 / \beta}\right\}\right)^{1 / 2}}-2 \frac{\delta}{\left(\log \left\{1+\left[-\alpha^{-1} \log (4 / 8)\right]^{-1 / \beta}\right\}\right)^{1 / 2}}+\frac{\delta}{\left(\log \left\{1+\left[-\alpha^{-1} \log (6 / 8)\right]^{-1 / \beta}\right\}\right)^{1 / 2}}}{\frac{\delta}{\left(\log \left\{1+\left[-\alpha^{-1} \log (2 / 8)\right]^{-1 / \beta}\right\}\right)^{1 / 2}}--\frac{\left(\log \left\{1+\left[-\alpha^{-1} \log (6 / 8)\right]^{-1 / \beta}\right\}\right)^{1 / 2}}{\left(\log ^{1 / 2}\right.}}
$$

Factorise (25) by factoring out $\delta$, since all the terms contain $\delta$ to have

$$
S=\frac{\left(\log \left\{1+\left[-\alpha^{-1} \log (2 / 8)\right]^{-1 / \beta}\right\}\right)^{-1 / 2}-2\left(\log \left\{1+\left[-\alpha^{-1} \log (4 / 8)\right]^{-1 / \beta}\right\}\right)^{-1 / 2}+\left(\log \left\{1+\left[-\alpha^{-1} \log (6 / 8)\right]^{-1 / \beta}\right\}\right)^{-1 / 2}}{\left(\log \left\{1+\left[-\alpha^{-1} \log (2 / 8)\right]^{-1 / \beta}\right\}\right)^{-1 / 2}-\left(\log \left\{1+\left[-\alpha^{-1} \log (6 / 8)\right]^{-1 / \beta}\right\}\right)^{-1 / 2}}
$$

The same procedure is also used to prove that the kurtosis does not contain parameter delta. Put (21) in (23) to have

$$
\begin{gathered}
K=\frac{\frac{\delta}{\left(\log \left\{1+\left[-\alpha^{-1} \log (1 / 8)\right]^{-1 / \beta}\right\}\right)^{1 / 2}}-\frac{\delta}{\left(\log \left\{1+\left[-\alpha^{-1} \log (3 / 8)\right]^{-1 / \beta}\right\}\right)^{1 / 2}}+\frac{\delta}{\left(\log \left\{1+\left[-\alpha^{-1} \log (5 / 8)\right]^{-1 / \beta}\right\}\right)^{1 / 2}}}{\frac{\delta}{\left(\log \left\{1+\left[-\alpha^{-1} \log (2 / 8)\right]^{-1 / \beta}\right\}\right)^{1 / 2}}--\frac{\delta}{\left(\log \left\{1+\left[-\alpha^{-1} \log (6 / 8)\right]^{-1 / \beta}\right\}\right)^{1 / 2}}} \\
-\frac{\frac{\left(\log \left\{1+\left[-\alpha^{-1} \log \left(\frac{7}{8}\right)\right]^{-\frac{1}{\beta}}\right\}\right)^{\frac{1}{2}}}{\delta}}{\left(\log \left\{1+\left[-\alpha^{-1} \log \left(\frac{2}{8}\right)\right]^{-\frac{1}{\beta}}\right\}\right)^{\frac{1}{2}}\left(\log \left\{1+\left[-\alpha^{-1} \log \left(\frac{6}{8}\right)\right]^{-\frac{1}{\beta}}\right\}\right)^{\frac{1}{2}}}
\end{gathered}
$$

Factorise (27) by factoring out $\delta$, since all the terms contain $\delta$ to have

$$
\begin{gathered}
K=\frac{\left(\log \left\{1+\left[-\alpha^{-1} \log (1 / 8)\right]^{-1 / \beta}\right\}\right)^{-1 / 2}-\left(\log \left\{1+\left[-\alpha^{-1} \log (3 / 8)\right]^{-1 / \beta}\right\}\right)^{-1 / 2}}{\left(\log \left\{1+\left[-\alpha^{-1} \log (2 / 8)\right]^{-1 / \beta}\right\}\right)^{-1 / 2}-\left(\log \left\{1+\left[-\alpha^{-1} \log (6 / 8)\right]^{-1 / \beta}\right\}\right)^{-1 / 2}} \\
\frac{\left(\log \left\{1+\left[-\alpha^{-1} \log (5 / 8)\right]^{-1 / \beta}\right\}\right)^{-1 / 2}-\left(\log \left\{1+\left[-\alpha^{-1} \log (7 / 8)\right]^{-1 / \beta}\right\}\right)^{-1 / 2}}{\left(\log \left\{1+\left[-\alpha^{-1} \log (2 / 8)\right]^{-1 / \beta}\right\}\right)^{-1 / 2}-\left(\log \left\{1+\left[-\alpha^{-1} \log (6 / 8)\right]^{-1 / \beta}\right\}\right)^{-1 / 2}}
\end{gathered}
$$

The results show that kurtosis and skewness are not a function of $\delta$ 
Table 1. The Galtons skewness and Moors kurtosis for some values of $(\alpha, \beta, \lambda, \delta)$ of the new WIR distribution.

\begin{tabular}{|c|c|c|c|c|c|c|c|c|c|}
\hline$\alpha$ & $\beta$ & $\delta$ & Skewness & Kurtosis & $\alpha$ & $\beta$ & $\delta$ & Skewness & Kurtosis \\
\hline 0.1 & 0.1 & 10 & 0.9366 & 6.2942 & 1 & 0.5 & 0.1 & 0.3182 & 1.2194 \\
\hline 0.1 & 0.1 & 20 & 0.9366 & 6.2942 & 1 & 1 & 1 & 0.1241 & 1.1021 \\
\hline 0.1 & 0.5 & 0.1 & 0.2781 & 1.1488 & 1 & 1 & 10 & 0.1241 & 1.1021 \\
\hline 0.1 & 0.5 & 10 & 0.2781 & 1.1488 & 1 & 10 & 1 & -0.0712 & 1.1933 \\
\hline 0.1 & 1 & 0.1 & 0.1061 & 1.0892 & 1 & 10 & 10 & -0.0712 & 1.1933 \\
\hline 0.1 & 1 & 0.5 & 0.1061 & 1.0892 & 1 & 20 & 0.5 & -0.0821 & 1.2050 \\
\hline 0.1 & 10 & 0.1 & -0.0713 & 1.1934 & 1 & 20 & 20 & -0.0821 & 1.2050 \\
\hline 0.1 & 10 & 1 & -0.0713 & 1.1934 & 10 & 0.1 & 0.1 & 0.1177 & 1.1704 \\
\hline 0.1 & 20 & 10 & -0.0821 & 1.2050 & 10 & 0.1 & 0.5 & 0.1177 & 1.1704 \\
\hline 0.1 & 20 & 20 & -0.0821 & 1.2050 & 10 & 0.5 & 0.5 & 0.1123 & 1.1589 \\
\hline 0.5 & 0.1 & 10 & 0.9407 & 6.3073 & 10 & 0.5 & 20 & 0.1123 & 1.1589 \\
\hline 0.5 & 0.1 & 20 & 0.9407 & 6.3073 & 10 & 1 & 0.1 & 0.0742 & 1.1307 \\
\hline 0.5 & 0.5 & 0.1 & 0.3176 & 1.1688 & 10 & 1 & 0.5 & 0.0742 & 1.1307 \\
\hline 0.5 & 0.5 & 0.5 & 0.3176 & 1.1688 & 10 & 10 & 1 & -0.0711 & 1.1933 \\
\hline 0.5 & 0.5 & 20 & 0.3176 & 1.1688 & 10 & 10 & 20 & -0.0711 & 1.1933 \\
\hline 0.5 & 1 & 1 & 0.1227 & 1.0921 & 10 & 20 & 0.1 & -0.0821 & 1.2050 \\
\hline 0.5 & 1 & 10 & 0.1227 & 1.0921 & 10 & 20 & 0.5 & -0.0821 & 1.2050 \\
\hline 0.5 & 1 & 20 & 0.1227 & 1.0921 & 20 & 0.5 & 1 & 0.0728 & 1.1463 \\
\hline 0.5 & 10 & 20 & -0.0712 & 1.1933 & 20 & 0.5 & 20 & 0.0728 & 1.1463 \\
\hline 0.5 & 10 & 0.1 & -0.0712 & 1.1933 & 20 & 1 & 0.1 & 0.0540 & 1.1347 \\
\hline 0.5 & 20 & 0.1 & -0.0821 & 1.2050 & 20 & 1 & 20 & 0.0540 & 1.1347 \\
\hline 0.5 & 20 & 20 & -0.0821 & 1.2050 & 20 & 10 & 0.1 & -0.0711 & 1.1933 \\
\hline 1 & 0.1 & 0.1 & 0.8983 & 6.6209 & 20 & 10 & 20 & -0.0711 & 1.1933 \\
\hline 1 & 0.1 & 0.5 & 0.8983 & 6.6209 & 20 & 20 & 0.1 & -0.0821 & 1.2050 \\
\hline 1 & 0.5 & 20 & 0.3182 & 1.2194 & 20 & 20 & 20 & -0.0821 & 1.2050 \\
\hline
\end{tabular}

Table 1 shows that the value of $\delta$ does not affect the shape of the WIR distribution. The skewness is only affected by the parameters $\alpha$ and $\beta$.

\subsection{Linear Representation}

Theorem 3: The PDF of WIR $(\boldsymbol{\alpha}, \boldsymbol{\beta}, \boldsymbol{\delta})$ is a linear function of the PDF of inverse Rayleigh distribution with parameter $\delta[\beta(k+1)+l]^{1 / 2}$.

\section{Proof.}

From the CDF in (8), we derive the PDF of WIR distribution as

$$
f_{X}(x)=\frac{2 \alpha \beta \delta^{2}}{x^{3}} \exp \left[-\beta(\delta / x)^{2}\right]\left\{1-\exp \left[-(\delta / x)^{2}\right]\right\}^{-\beta-1} \times \exp \left\{-\alpha\left[\frac{\exp \left[-(\delta / x)^{2}\right]}{1-\exp \left[-(\delta / x)^{2}\right]}\right]^{\beta}\right\}
$$

and using Taylor series expansion of

$$
\exp \left\{-\alpha\left[\frac{\exp \left[-(\delta / x)^{2}\right]}{1-\exp \left[-(\delta / x)^{2}\right]}\right]^{\beta}\right\}=\sum_{k=0}^{\infty} \frac{(-1)^{k} \alpha^{k}}{k !}\left[\frac{\exp \left[-(\delta / x)^{2}\right]}{1-\exp \left[-(\delta / x)^{2}\right]}\right]^{k \beta} .
$$

from [17], we have the following series if (28) is substituted into (27) to have

$$
f_{X}(x)=\sum_{k=0}^{\infty} \frac{(-1)^{k} \alpha^{k}}{k !} \frac{2 \alpha \beta \delta^{2}}{x^{3}} \exp \left[-\beta(k+1)(\delta / x)^{2}\right]\left\{1-\exp \left[-(\delta / x)^{2}\right]\right\}^{-[\beta(k+1)+1]}
$$

Then, further expansion of (29) gives 


$$
f_{X}(x)=\sum_{k, l=0}^{\infty} \frac{(-1)^{k} \alpha^{k+1} \beta}{k !}(-[\beta(k+1)+1]) \frac{2 \delta^{2}}{x^{3}} \exp \left[-[\beta(k+1)+l](\delta / x)^{2}\right]
$$

Hence the linear representation of the new WIR distribution is given as

$$
f(x, \alpha, \beta, \delta)=\sum_{k, l=0}^{\infty} \xi_{k, l} h_{\beta(k+1)+l-1}(x)
$$

where

$$
\xi_{k, l}=\sum_{k, l=0}^{\infty} \frac{(-1)^{k} \beta \alpha^{k+1} \Gamma(\beta(k+1)+l+1)}{k ! l ![\beta(k+1)+l-1] \Gamma(\beta(k+1)+1)}
$$

and

$$
h_{\beta(k+1)+l}(x)=\frac{2[\beta(k+1)+1] \delta^{2}}{x^{3}} \exp \left[-[\beta(k+1)+l](\delta / x)^{2}\right]
$$

where $h_{\beta(k+1)+l}(x)$ is the PDF of Inverse Rayleigh distribution with scale parameter $\delta[\beta(k+1)+l]^{1 / 2}$

\subsection{The Order Statistic}

The $n$th order statistic of a random variable $X$ is given by

$$
f_{r: n}=\frac{n !}{(r-1) !(n-r) !} f_{X}(x)\left[F_{X}(x)\right]^{(r-1)}\left[1-F_{X}(x)\right]^{(n-r)}
$$

If $X \sim \operatorname{WIR}(\alpha, \beta, \delta)$, then the $f_{r: n}\left(x_{r}\right)$ of $X$ is derived thus;

$$
\begin{aligned}
f_{r: n} & =\frac{n !}{\pi(r-1) !(n-r) !} \sum_{j}^{\infty}(-1)^{j}\left(\begin{array}{l}
n-r \\
j
\end{array}\right) f_{X}(x)\left[F_{X}(x)\right]^{(j+r-1)} \\
{\left[F_{X}(x)\right]^{(j+r-1)} } & =\left(1-\exp \left\{-\alpha\left[\frac{\exp \left[-(\delta / x)^{2}\right]}{1-\exp \left[-(\delta / x)^{2}\right]}\right]^{\beta}\right\}\right)^{(j+r-1)} \\
{\left[F_{X}(x)\right]^{(j+r-1)} } & =\sum_{m}^{\infty}(-1)^{m}\left(\begin{array}{l}
j+r-1 \\
m
\end{array}\right) \exp \left\{-m \alpha\left[\frac{\exp \left[-(\delta / x)^{2}\right]}{1-\exp \left[-(\delta / x)^{2}\right]}\right]^{\beta}\right\}
\end{aligned}
$$

Inserting (33) and (34) into (32) gives

$$
\begin{gathered}
\frac{n !}{\pi(r-1) !(n-r) !} \sum_{j, m}^{\infty}(-1)^{j+m}\left(\begin{array}{c}
n-r \\
j
\end{array}\right)\left(\begin{array}{c}
j+r-1 \\
m
\end{array}\right) \frac{2 \alpha \beta \delta^{2}}{x^{3}} \exp \left[-\beta(\delta / x)^{2}\right]\left\{1-\exp \left[-(\delta / x)^{2}\right]\right\}^{-\beta-1} \times \exp \left\{\begin{array}{c}
f_{r: n}= \\
-\alpha(m+ \\
\text { 1) } \left.\left[\frac{\exp \left[-(\delta / x)^{2}\right]}{1-\exp \left[-(\delta / x)^{2}\right]}\right]^{\beta}\right\}
\end{array}\right.
\end{gathered}
$$

By series expansion, we have

$$
\exp \left\{-\alpha(m+1)\left[\frac{\exp \left[-(\delta / x)^{2}\right]}{1-\exp \left[-(\delta / x)^{2}\right]}\right]^{\beta}\right\}=\sum_{p=0}^{\infty} \frac{(-1)^{p} \alpha^{k}(m+1)^{p}}{p !} \exp \left[-p \beta(\delta / x)^{2}\right]\left\{1-\exp \left[-(\delta / x)^{2}\right]\right\}^{-p \beta}
$$

Thus,

$$
\begin{gathered}
f_{r: n}=\frac{n !}{(r-1) !(n-r) !} \sum_{j, m, p}^{\infty}(-1)^{j+m+p} \frac{\alpha^{p}(m+1)^{p}}{p !}\left(\begin{array}{c}
n-r \\
j
\end{array}\right)\left(\begin{array}{c}
j+r-1 \\
m
\end{array}\right) \frac{2 \alpha \beta \delta^{2}}{x^{3}} \exp \left[-\beta(p+1)(\delta / x)^{2}\right] \\
\left\{1-\exp \left[-(\delta / x)^{2}\right]\right\}^{-[\beta(p+1)+1}
\end{gathered}
$$

Further series expansion of (37) gives

$$
f_{r: n}=\frac{n !}{(r-1) !(n-r) !} \sum_{j, m, p=0}^{\infty}(-1)^{j+m+p} \frac{\alpha^{p}(m+1)^{p}}{p !}\left(\begin{array}{l}
n-r \\
j
\end{array}\right)\left(\begin{array}{l}
j+r-1 \\
m
\end{array}\right)\left(\begin{array}{l}
\beta(p+1)+1 \\
q
\end{array}\right) .
$$

Let

$$
\omega_{m, p}=\frac{n !}{(r-1) !(n-r) !} \sum_{j, m, p=0}^{\infty}(-1)^{j+m+p} \frac{\alpha^{p}(m+1)^{p}}{p !}\left(\begin{array}{c}
n-r \\
j
\end{array}\right)\left(\begin{array}{c}
j+r-1 \\
m
\end{array}\right)\left(\begin{array}{c}
\beta(p+1)+1 \\
q
\end{array}\right)
$$

and

$$
h_{\beta(p+1)+q}=\frac{2 \alpha \beta \delta^{2}}{x^{3}} \exp \left[-[\beta(p+1)+q](\delta / x)^{2}\right]
$$


Therefore, the density function of order statistic is given as

$$
f_{r: n}(x, \alpha, \beta, \delta)=\omega_{m, p} h_{\beta(p+1)+q}(x)
$$

\subsection{The Monotone Likelihood Ratio Property of WIR Distribution}

In statistics, the monotone likelihood ratio property (MLRP) is a property of the ratio of two probability density functions.

Definition 4. Let $\boldsymbol{f}_{\boldsymbol{X}}(\boldsymbol{x})$ and $\boldsymbol{g}_{\boldsymbol{X}}(\boldsymbol{x})$ be the PDFs of two distributions with respect to $\boldsymbol{x}$, iffor every $\boldsymbol{x}_{\mathbf{2}}>\boldsymbol{x}_{\mathbf{1}}, \boldsymbol{x}_{\mathbf{1}}, \boldsymbol{x}_{\mathbf{2}} \exists$ $\boldsymbol{x}$, then

$$
\frac{f_{X}\left(x_{2}\right)}{g_{X}\left(x_{2}\right)} \geq \frac{f_{X}\left(x_{1}\right)}{g_{X}\left(x_{1}\right)}
$$

that is if the ratio is non-decreasing in the argument $x$.

If the functions are first-differentiable, the property is sometimes stated as

$$
\frac{\partial}{\partial x}\left(\frac{f_{X}(x)}{g_{X}(x)}\right) \geq 0
$$

Then $f_{X}(x)$ and $g_{X}(x)$ have the MLRP in $x$.

For two distributions that satisfy the definition with respect to some argument $x$, we say they "have the MLRP in $x$.

\subsection{The Moment}

Theorem 4: If $\boldsymbol{X} \sim W-I R(\boldsymbol{\alpha}, \boldsymbol{\beta}, \boldsymbol{\delta})$, the $\boldsymbol{r}$ th moment of $\boldsymbol{X}$ exist only for $\boldsymbol{r} \leq \mathbf{1}$ and it is given by

$$
E\left(X^{r}\right)=\sum_{k, l=0}^{\infty} \frac{(-1)^{k+1} \alpha^{k+1} \beta}{k !}\left(\begin{array}{c}
-[\beta(k+1)+1] \\
l
\end{array}\right) \frac{\delta^{r} \Gamma(1-r / 2)}{[\beta(k+1)+l]^{1-r / 2}}
$$

\section{Proof.}

Recall the linear expansion of the PDF of WIR distribution in (30) as

$$
f_{X}(x)=\sum_{k, l=0}^{\infty} \frac{(-1)^{k} \alpha^{k+1} \beta}{k !}\left(\begin{array}{c}
-[\beta(k+1)+1] \\
l
\end{array}\right) \frac{2 \delta^{2}}{x^{3}} \exp \left[-[\beta(k+1)+l](\delta / x)^{2}\right]
$$

The $r$ th moment of a random variable $X$ is given by $E\left(X^{r}\right)=\int x f_{X}(x) d x$. So, that we have

$$
E\left(X^{r}\right)=\int_{0}^{\infty} x^{r} \sum_{k, l=0}^{\infty} \frac{(-1)^{k} \alpha^{k+1} \beta}{k !}\left(\begin{array}{c}
-[\beta(k+1)+1] \\
l
\end{array}\right) \frac{2 \delta^{2}}{x^{3}} \exp \left[-[\beta(k+1)+l](\delta / x)^{2}\right] d x
$$

The sum of integral is equal to the integral of a sum so that we have

$$
E\left(X^{r}\right)=\sum_{k, l=0}^{\infty} \frac{(-1)^{k} \alpha^{k+1} \beta}{k !}\left(\begin{array}{c}
-[\beta(k+1)+1] \\
l
\end{array}\right) \int_{0}^{\infty} x^{r} \frac{2 \delta^{2}}{x^{3}} \exp \left[-[\beta(k+1)+l](\delta / x)^{2}\right] d x
$$

Let $w=\frac{\delta^{2}}{x^{2}}$ and $x=\frac{\delta}{\sqrt{w}}$, so that $d x=\frac{-x^{3} d w}{2 \delta^{2}}$. Equation (42) becomes

$$
E\left(X^{r}\right)=\sum_{k, l=0}^{\infty} \frac{(-1)^{k+1} \alpha^{k+1} \beta}{k !}(-[\beta(k+1)+1]) \delta^{r} \int_{0}^{\infty} w^{-\frac{r}{2}} \exp [-[\beta(k+1)+l] w] d w
$$

Using the gamma function on (43) gives

$$
E\left(X^{r}\right)=\sum_{k, l=0}^{\infty} \frac{(-1)^{k+1} \alpha^{k+1} \beta}{k !}(-[\beta(k+1)+1]) \frac{\delta^{r} \Gamma(1-r / 2)}{[\beta(k+1)+l]^{1-r / 2}}
$$

Equation (44) completes the proof and it is the $r$ th moment of WIR distribution with parameters $\alpha, \beta$, and $\delta$. If $k=l=0$, the $r$ th moment becomes

$$
E\left(X^{r}\right)=\alpha(\beta+1) \beta^{r / 2} \delta^{r} \Gamma(1-r / 2)
$$

The mean of WIR distribution is derived from (44) if $r=1$ and it is given by

$$
E(X)=\sum_{k, l=0}^{\infty} \frac{(-1)^{k+1} \alpha^{k+1} \beta}{k !}\left(\begin{array}{c}
-[\beta(k+1)+1] \\
l
\end{array}\right) \frac{\delta \sqrt{\pi}}{[\beta(k+1)+l]^{1 / 2}}
$$


According to [18], to have a reduced form of the moment, we set $k=l=0$, the mean in (46) is then reduced to

$$
E(X)=\alpha \delta(\beta+1) \sqrt{\beta \pi}
$$

It is obvious that only the first moment and fractional moment exist. Higher moments of W-IR distribution do not exist. Thus, its variance does not exist.

\subsection{The Asymptotes of WIR Distribution}

The value $x$ of the function WIR distribution when $f_{X}(x)$ approaches $\infty$.

$$
\lim _{x \rightarrow \infty} f_{X}(x)=\infty
$$

The functions $f_{X}(x)$ and $h_{X}(x)$ of WIR distribution will be undefined if

$$
\left\{1-\exp \left[-(\delta / x)^{2}\right]\right\}=0
$$

and $x=\infty$. Thus, the vertical asymptote of $f_{X}(x)$ is given by

$$
\lim _{x \rightarrow \infty} f_{X}(x)=\infty
$$

and the vertical asymptote of $h_{X}(x)$ is given by

$$
\lim _{x \rightarrow \infty} h_{X}(x)=\infty
$$

So, equations (49) and (50) are the vertical asymptotes of the PDF and hazard function of WIR distribution respectively.

\section{Estimation}

\subsection{The Maximum Likelihood Estimation}

For a random sample $\mathrm{x}=\left(x_{1}, x_{2}, \ldots, x_{n}\right)$ of size $n$ from (10) the likelihood function is

$$
L(\alpha, \beta, \delta)=2^{n} \alpha^{n} \beta^{n} \delta^{2 n} e^{-\beta \sum\left(\frac{\delta}{x}\right)^{2}} \sum\left(\frac{1}{x^{3}}\right) e^{-\alpha \Sigma\left(e^{\left.\frac{\delta}{x}\right)^{2}-1}\right)^{\beta-1}} \Pi\left(1-e^{-\left(\frac{\delta}{x}\right)^{2}}\right)^{-\beta-1}
$$

hence, the $\log$-likelihood function $l(\alpha, \beta, \delta)=\log L(\alpha, \beta, \delta)$ becomes

$$
\begin{gathered}
l(\alpha, \beta, \delta)=n \ln (2)+n \ln (\alpha)+n \ln (\beta)+2 n \ln (\delta)-3 \sum \ln (x)-\beta \sum(\delta / x)^{2}-(\beta+1) \sum\left(1-\exp \left(-(\delta / x)^{2}\right)\right)- \\
\alpha \sum\left(e^{\left.\frac{\delta}{x}\right)^{2}-1}\right)^{\beta-1}
\end{gathered}
$$

Differentiating the log-likelihood function $l(\alpha, \beta, \delta)$ partially with respect to $\alpha, \beta$, and $\delta$

$$
\begin{gathered}
\frac{\partial l(\alpha, \beta, \delta)}{\partial \alpha}=\frac{n}{\alpha}-\sum\left(e^{\left.\left(\frac{\delta}{x}\right)^{2}-1\right)^{-\beta}}\right) \\
\frac{\partial l(\alpha, \beta, \delta)}{\partial \beta}=\frac{n}{\beta}-\sum\left(\frac{\delta}{x}\right)^{2}-\sum\left(1-e^{-\left(\frac{\delta}{x}\right)^{2}}\right)+\sum\left(e^{\left(\frac{\delta}{x}\right)^{2}}-1\right)^{-\beta} \ln \left(e^{\left(\frac{\delta}{x}\right)^{2}}-1\right) \\
\frac{\partial l(\alpha, \beta, \delta)}{\partial \delta}=\frac{2 n}{\delta}+2 \beta \delta \sum\left(\frac{\left(e^{\left(\frac{\delta}{x}\right)^{2}}-1\right)^{-\beta} e^{\left(\frac{\delta}{x}\right)^{2}}}{x^{2}\left(e^{\left(\frac{\delta}{x}\right)^{2}}-1\right)}\right)-2 \beta \delta \sum\left(\frac{1}{x^{2}}\right)-2(\beta+1) \delta \sum\left(\frac{e^{\left(-\frac{\delta}{x}\right)^{2}}}{x^{2}}\right)
\end{gathered}
$$

solving for $\alpha, \beta$ and $\delta$ from equations (57) - (59) analytically by equating to zero is impossible. The maximum likelihood estimator of $\alpha, \beta$ and $\delta$ says $\hat{\alpha}, \hat{\beta}$ and $\hat{\delta}$ are the solutions to the equations (57) - (59). Thus, the solution is intractable, therefore, the numerical method of Newton Raphson is adopted as follows:

$$
L_{11}=\frac{\partial^{2} l(\alpha, \beta, \delta)}{\partial \alpha^{2}}=-\frac{n}{\alpha^{2}}
$$




$$
\begin{aligned}
& L_{22}=\frac{\partial^{2} l(\alpha, \beta, \delta)}{\partial \beta^{2}}=-\frac{n}{\beta^{2}}-\alpha \sum\left(e^{\left(\frac{\delta}{x}\right)^{2}}-1\right)^{-\beta} \ln \left(e^{\left(\frac{\delta}{x}\right)^{2}}-1\right)^{2} \\
& L_{12}=\frac{\partial^{2} l(\alpha, \beta, \delta)}{\partial \alpha \partial \beta}=\sum\left(e^{\left(\frac{\delta}{x}\right)^{2}}-1\right)^{-\beta} \ln \left(e^{\left(\frac{\delta}{x}\right)^{2}}-1\right) \\
& L_{13}=\frac{\partial^{2} l(\alpha, \beta, \delta)}{\partial \alpha \partial \delta}=2 \beta \delta \sum\left(\frac{\left(e^{\left(\frac{\delta}{x}\right)^{2}}-1\right)^{-\beta} e^{\left(\frac{\delta}{x}\right)^{2}}}{x^{2}\left(e^{\left(\frac{\delta}{x}\right)^{2}}-1\right)}\right) \\
& L_{23}=\frac{\partial^{2} l(\alpha, \beta, \delta)}{\partial \beta \partial \delta}=-2 \sum\left(\frac{e^{-\left(\frac{\delta}{x}\right)^{2}}}{x}\right)+2 \alpha \delta \sum\left(\frac{\left(e^{\left(\frac{\delta}{x}\right)^{2}}-1\right)^{-\beta} e^{\left(\frac{\delta}{x}\right)^{2}}}{x^{2}\left(e^{\left(\frac{\delta}{x}\right)^{2}}-1\right)}\right)-2 \alpha \beta \delta \sum\left(\frac{\left(e^{\left(\frac{\delta}{x}\right)^{2}}-1\right)^{-\beta} e^{\left(\frac{\delta}{x}\right)^{2}} \ln \left(e^{\left(\frac{\delta}{x}\right)^{2}}-1\right)}{x^{2}\left(e^{\left(\frac{\delta}{x}\right)^{2}}-1\right)}\right) \\
& L_{33}=\frac{\partial^{2} l(\alpha, \beta, \delta)}{\partial \delta^{2}}= \\
& -\frac{2 n}{\delta^{2}}-2 \beta \sum\left(\frac{1}{x^{2}}\right)-2(\beta+1) \sum\left(\frac{e^{-\left(\frac{\delta}{x}\right)^{2}}}{x^{4}}\right)-4 \alpha \beta^{2} \delta^{2} \sum\left(\frac{\left(e^{\left(\frac{\delta}{x}\right)^{2}}-1\right)^{-\beta} e^{\left(\frac{\delta}{x}\right)^{2}}}{x^{4}\left(e^{\left(\frac{\delta}{x}\right)^{2}}-1\right)}\right)+4(\beta+1) \delta^{2} \sum\left(\frac{e^{\left(\frac{\delta}{x}\right)^{2}}}{x^{4}\left(e^{\left(\frac{\delta}{x}\right)^{2}}-1\right)}\right)+ \\
& 2 \alpha \beta \sum\left(\frac{\left(e^{\left(\frac{\delta}{x}\right)^{2}}-1\right)^{-\beta} e^{\left(\frac{\delta}{x}\right)^{2}}}{x^{2}\left(e^{\left(\frac{\delta}{x}\right)^{2}}-1\right)}\right)+4 \alpha \delta^{2} \sum\left(\frac{\left(e^{\left(\frac{\delta}{x}\right)^{2}}-1\right)^{-\beta} e^{\left(\frac{\delta}{x}\right)^{2}}}{x^{4}\left(e^{\left(\frac{\delta}{x}\right)^{2}}-1\right)}\right)-4 \alpha \beta \delta^{2} \sum\left(\frac{\left(e^{\left(\frac{\delta}{x}\right)^{2}}-1\right)^{-\beta}\left(e^{\left(\frac{\delta}{x}\right)^{2}}\right)^{2}}{x^{4}\left(e^{\left(\frac{\delta}{x}\right)^{2}}-1\right)^{2}}\right)
\end{aligned}
$$

Where the elements of the Fisher information matrix are expressed in (60) - (65). Hence,

$$
J_{k}=\left[\begin{array}{ccc}
\frac{\partial^{2} l(\alpha, \beta, \delta)}{\partial \alpha^{2}} & \frac{\partial^{2} l(\alpha, \beta, \delta)}{\partial \alpha \partial \beta} & \frac{\partial^{2} l(\alpha, \beta, \delta)}{\partial \alpha \partial \delta} \\
& \frac{\partial^{2} l(\alpha, \beta, \delta)}{\partial \beta^{2}} & \frac{\partial^{2} l(\alpha, \beta, \delta)}{\partial b e t a \partial \delta} \\
& & \frac{\partial^{2} l(\alpha, \beta, \delta)}{\partial \delta^{2}}
\end{array}\right]
$$

The Jacobian matrix must be a non-singular symmetric matrix so its inverse must exist. So, using the Newton Raphson method we have

$$
\left[\begin{array}{l}
\alpha_{k+1} \\
\beta_{k+1} \\
\delta_{k+1}
\end{array}\right]=\left[\begin{array}{l}
\alpha_{k} \\
\beta_{k} \\
\delta_{k}
\end{array}\right]-J_{k}{ }^{-1}\left[\begin{array}{c}
\frac{\partial l(\alpha, \beta, \delta)}{\partial \alpha} \\
\frac{\partial l(\alpha, \beta, \delta)}{\partial \beta} \\
\frac{\partial l(\alpha, \beta, \delta)}{\partial \delta}
\end{array}\right]
$$

with error term $\varepsilon$ being the absolute differences between the new and the previous value of $\alpha$ and $\lambda$ in the iterative algorithm. That is

$$
\left.\left[\begin{array}{l}
\varepsilon_{k+1}(\alpha) \\
\varepsilon_{k+1}(\beta) \\
\varepsilon_{k+1}(\delta)
\end{array}\right]=\left[\begin{array}{l}
\alpha_{k+1} \\
\beta_{k+1} \\
\delta_{k+1}
\end{array}\right]-\left[\begin{array}{l}
\alpha_{k} \\
\beta_{k} \\
\delta_{k}
\end{array}\right]\right]
$$

where $\alpha_{k}, \beta_{k}$ and $\delta_{k}$ are the initial values of $\alpha, \beta$, and $\delta$ respectively.

\subsection{The Quantile Function Estimation Method}

This method is a method that is not common. For three parameters distribution, we have the system of equations given by 


$$
\begin{aligned}
Q_{1} & =\frac{\delta}{\left(\log \left\{1+\left[-\alpha^{-1} \log (3 / 4)\right]^{-1 / \beta}\right\}\right)^{1 / 2}} \\
Q_{2} & =\frac{\delta}{\left(\log \left\{1+\left[-\alpha^{-1} \log (1 / 2)\right]^{-1 / \beta}\right\}\right)^{1 / 2}} \\
Q_{3} & =\frac{\delta}{\left(\log \left\{1+\left[-\alpha^{-1} \log (1 / 4)\right]^{-1 / \beta}\right\}\right)^{1 / 2}}
\end{aligned}
$$

where $Q_{1}, Q_{2}$ and $Q_{3}$ are the 1 st, 2 nd and 3rd quartiles respectively, which can be obtained from the given data. Thus, we solve the system of equations for the parameters $\alpha, \beta$ and $\delta$, which gives the estimates of the three parameters, the method was successfully implemented by [19].

\subsection{The Bayesian Estimation}

In probability and statistics, the Bayesian inference is a statistical inference method in which Bayes' theorem is used to update the probability for a hypothesis as more evidence or facts from data becomes available. It is an important technique in mathematical statistics. It employs the use of the posterior predictive distribution to do predictive inference. Several methods of Bayesian estimation select measurements of central tendency from the posterior distribution [20]. For example, if there exists a finite mean for the posterior distribution, then the posterior mean is a method of estimation [21].

Bayesian inference has been applied in different Bioinformatics scenarios, by building inferences from prior knowledge and available data, such as differential gene expression analysis and general cancer risk model (Continuous Individualized Risk Index), where serial measurements are incorporated to update a Bayesian model [22].

In this section, we consider three types of loss functions namely: Squared Error Loss Function which is also known as Quadratic Loss Function, the Linex Loss Function, and the Generalization of Entropy Loss Function. Therefore, the Bayesian estimators of the parameters of the new WIR distribution were considered under these three loss functions.

\subsubsection{The Prior Distribution}

To obtain the estimates of $\alpha, \beta$, and $\delta$, we assumed that all the parameters are real-valued random variables with probability density function $\pi(\alpha, \beta, \delta)$. The posterior distribution $\operatorname{Pr}(\alpha, \beta, \delta \mid x)$ is the conditional probability density function of $\alpha, \beta$, and $\delta$ given data $\mathrm{x}$. We assumed that $\alpha, \beta$ and $\delta$ are unknown and certainly their conjugate priors do not exist. So, we consider the following prior distribution on $\alpha, \beta$, and $\delta$; An informative prior distribution of the form $\pi(\beta) \sim G\left(a_{2}, b_{2}\right)$ is assumed for $\beta$ while non-informative priors of the forms $\pi(\delta) \sim U(0, \delta)$ and $\pi(\alpha) \sim U(0, \alpha)$ are assumed for both parameters $\delta$ and $\alpha$.

$$
\begin{gathered}
\pi(\delta)=\frac{1}{\delta} \quad \delta>0 \\
\pi(\alpha)=\frac{1}{\alpha} \quad \alpha>0 \\
\pi(\beta)=\frac{a^{b}}{\Gamma(b)} \alpha^{b-1} e^{-a \beta} \\
a>0, b>0, \beta>0
\end{gathered}
$$

Then, the joint prior distribution on $\alpha, \beta$ and $\delta$ can be expressed as

$$
\pi(\alpha, \beta, \delta)=\frac{b^{a}}{\alpha \delta \gamma(a)} \beta^{a-1} e^{-b \beta} \quad \alpha>0, \beta>0, a>0, b>0, \delta>0
$$

\subsubsection{The Posterior Analysis}

Based on the sample $x_{1}, x_{2}, \ldots, x_{n}$ and using the joint prior distribution in (69) and the likelihood in (55) the joint posterior distribution of $\alpha, \beta$, and $\delta$ can be expressed as

$$
\operatorname{Pr}(\alpha, \beta, \delta \mid x) \propto \frac{\pi(\alpha, \beta, \delta) l(\alpha, \beta, \delta)}{\iiint \pi(\alpha, \beta, \delta) l(\alpha, \beta, \delta) \partial(\alpha, \beta, \delta)}
$$

which results in

$$
\left.\operatorname{Pr}(\alpha, \beta, \delta \mid x)=\frac{2^{n} \kappa \alpha^{n-1} \beta^{n+a-1} \delta^{2 n-1}}{\alpha \delta \Gamma(a)} e^{-\beta\left(b+\sum\left(\frac{\delta}{x}\right)^{2}\right)} \sum\left(\frac{1}{x^{3}}\right)\right) e^{-\alpha \Sigma\left(e^{\left.\frac{\delta}{x}\right)^{2}-1}\right)^{\beta-1}} \Pi\left(1-e^{-\left(\frac{\delta}{x}\right)^{2}}\right)^{-\beta-1}
$$




$$
\left.\kappa=\iiint \frac{2^{n} \kappa \alpha^{n-1} \beta^{n+a-1} \delta^{2 n-1}}{\alpha \delta \Gamma(a)} e^{-\beta\left(b+\sum\left(\frac{\delta}{x}\right)^{2}\right)} \sum\left(\frac{1}{x^{3}}\right)\right) e^{-\alpha \Sigma\left(e^{\left.\frac{\delta}{x}\right)^{2}-1}\right)^{\beta-1}} \Pi\left(1-e^{-\left(\frac{\delta}{x}\right)^{2}}\right)^{-\beta-1} \partial \alpha \partial \delta \partial \beta
$$

\subsubsection{The Lindley's Approximation}

The joint posterior distribution of the unknown parameters $\alpha, \beta$, and $\delta$ cannot be solved analytically because it has no closed-form solution. Therefore, in this study we employed the Lindley's approximation method to investigate the quantities of the parameters $\alpha, \beta$, and $\delta$ under the Squared error loss function, Linex loss function, and the General Entropy loss function. According to [23], if $n$ is sufficiently large, any ratio of the integral of the form

$$
I(x)=E[u(\alpha, \beta, \delta)]=\frac{\iiint u(\alpha, \beta, \delta) e^{l(\alpha, \beta, \delta)+\rho(\alpha, \beta, \delta)} \partial(\alpha, \beta, \delta)}{\iiint e^{l(\alpha, \beta, \delta)+\rho(\alpha, \beta, \delta)} \partial(\alpha, \beta, \delta)}
$$

where $u(\alpha, \beta, \delta)$ is a function of $\alpha, \beta$ and $\delta$ only, $l(\alpha, \beta, \delta)$ is the log-likelihood and $\rho(\alpha, \beta, \delta)$ is the $\log$ of prior distribution $\pi(\alpha, \beta, \delta)$. Equation (72) can be evaluated as

$$
\begin{gathered}
I(x)=u(\hat{\alpha}, \hat{\beta}, \hat{\delta})+\left(u_{1} a_{1}+u_{2} a_{2}+u_{3}+a_{3}+a_{4}+a_{5}\right)+\frac{1}{2} A\left(u_{1} \sigma_{11}+u_{2} \sigma_{12}+u_{3} \sigma_{13}\right)+\frac{1}{2} B\left(u_{1} \sigma_{21}+u_{2} \sigma_{22}+\right. \\
\left.u_{3} \sigma_{23}\right)+\frac{1}{2} C\left(u_{1} \sigma_{31}+u_{2} \sigma_{32}+u_{3} \sigma_{33}\right)
\end{gathered}
$$

where $\hat{\alpha}, \hat{\beta}$ and $\hat{\delta}$ are the maximum likelihood estimators of $\alpha, \beta$ and $\delta$ while

$$
\begin{gathered}
a_{i}=\rho_{1} \sigma_{i 1}+\rho_{2} \sigma_{i 2}+\rho_{3} \sigma_{i 3} \quad i=1,2,3 . \\
a_{4}=u_{12} \sigma_{12}+u_{13} \sigma_{13}+u_{23} \sigma_{23} \\
a_{5}=\frac{1}{2}\left(u_{11} \sigma_{11}+u_{22} \sigma_{22}+u_{33} \sigma_{33}\right) \\
A=\sigma_{11} \mathrm{t}_{11}+2 \sigma_{12} L_{121}+2 \sigma_{13} L_{131}+2 \sigma_{23} L_{231}+\sigma_{22} L_{221}+\sigma_{33} L_{221} \\
B=\sigma_{11} L_{112}+2 \sigma_{12} L_{122}+2 \sigma_{13} L_{132}+2 \sigma_{23} L_{232}+\sigma_{22} L_{222}+\sigma_{33} L_{332} \\
C=\sigma_{11} L_{113}+2 \sigma_{12} L_{123}+2 \sigma_{13} L_{133}+2 \sigma_{23} L_{233}+\sigma_{22} L_{223}+\sigma_{33} L_{333} \\
\rho_{i}=i=1,2,3 \\
u_{i}=\frac{\partial u\left(\theta_{1}, \theta_{2}, \theta_{3}\right)}{\partial \theta_{i}} \quad i=1,2,3 \\
u_{i, j}=\frac{\partial^{2} u\left(\theta_{1}, \theta_{2}, \theta_{3}\right)}{\partial \theta_{i} \partial \theta_{j}} \quad i, j=1,2,3 \\
L_{i, j, k}=\frac{\partial^{3} l\left(\theta_{1}, \theta_{2}, \theta_{3}\right)}{\partial \theta_{i} \partial \theta_{j} \partial \theta_{k}} \quad i, j, k=1,2,3
\end{gathered}
$$

with $\theta_{1}=\alpha, \theta_{2}=\beta$ and $\theta_{3}=\delta . \sigma_{i, j}$ is the $(i, j)^{t h}$ element of the inverse of the matrix $L_{i, j}$ all evaluated at the maximum likelihood estimates.

From (69),

$$
\rho=a \ln b-\ln \alpha-\ln \beta-\ln \delta+(a-1) \ln \beta-b \beta
$$

so, therefore,

$$
\rho_{1}=-\frac{1}{\alpha}, \rho_{2}=\frac{a-1}{\beta}-b, \rho_{3}=-\frac{1}{\delta}
$$

$L_{11}, L_{12}=L_{21}, L_{13}=L_{31}, L_{22}, L_{23}=L_{32}$ and $L_{33}$ have been expressed in equations (60) - (65)

$$
\begin{gathered}
L_{111}=\frac{2 n}{\alpha^{3}} \\
L_{112}=L_{121}=L_{211}=0 \\
L_{113}=L_{131}=L_{311}=0 \\
L_{222}=\frac{2 n}{\beta^{3}}+\alpha \sum\left(e^{\left(\frac{\delta}{x}\right)^{2}}-1\right)^{-\beta} \ln \left(e^{\left(\frac{\delta}{x}\right)^{2}}-1\right)^{3}
\end{gathered}
$$




$$
\begin{aligned}
& L_{123}=L_{132}=L_{321}=L_{231}=L_{312}=L_{213}= \\
& =-2 \beta \delta \sum\left(\frac{e^{\left(\frac{\delta}{x}\right)^{2}}\left(e^{\left(\frac{\delta}{x}\right)^{2}}-1\right)^{-\beta} \ln \left(e^{\left(\frac{\delta}{x}\right)^{2}}-1\right)}{x^{2}\left(e^{\left(\frac{\delta}{x}\right)^{2}}-1\right)}\right)+2 \delta \sum\left(\frac{\left(e^{\left(\frac{\delta}{x}\right)^{2}}-1\right)^{-\beta} e^{\left(\frac{\delta}{x}\right)^{2}}}{x^{2}\left(e^{\left(e^{2}\right)^{2}}-1\right)}\right) \\
& L_{122}=L_{221}=L_{212}=-\sum\left(e^{\left(\frac{\delta}{x}\right)^{2}}-1\right)^{-\beta} \ln \left(e^{\left(\frac{\delta}{x}\right)^{2}}-1\right)^{2} \\
& L_{133}=L_{331}=L_{313}=-4 \beta^{2} \delta^{2} \sum\left(\frac{\left(e^{\left(\frac{\delta}{x}\right)^{2}}-1\right)^{-\beta} e^{\left(\frac{\delta}{x}\right)^{2}}}{x^{4}\left(e^{\left(\frac{\delta}{x}\right)^{2}}-1\right)^{2}}\right)+2 \beta \sum\left(\frac{\left(e^{\left(\frac{\delta}{x}\right)^{2}}-1\right)^{-\beta} e^{\left(\frac{\delta}{x}\right)^{2}}}{x^{2}\left(e^{\left(\frac{\delta}{x}\right)^{2}}-1\right)}\right)+ \\
& +4 \beta \delta^{2} \sum\left(\frac{\left(e^{\left(\frac{\delta}{x}\right)^{2}}-1\right)^{-\beta} e^{\left(\frac{\delta}{x}\right)^{2}}}{x^{4}\left(e^{\left(e^{\frac{\delta}{x}}\right)^{2}}-1\right)}\right)-4 \beta \delta^{2} \sum\left(\frac{\left(e^{\left(\frac{\delta}{x}\right)^{2}}-1\right)^{-\beta}\left(e^{\left(\frac{\delta}{x}\right)^{2}}\right)^{2}}{x^{4}\left(e^{\left(\frac{\delta}{x}\right)^{2}}-1\right)^{2}}\right) \\
& L_{223}=L_{232}=L_{322}=2 \alpha \beta \delta \sum\left(\frac{\left(e^{\left(\frac{\delta}{x}\right)^{2}}-1\right)^{-\beta} e^{\left(\frac{\delta}{x}\right)^{2}} \ln \left(e^{\left(\frac{\delta}{x}\right)^{2}}-1\right)^{2}}{x^{2}\left(e^{\left(\frac{\delta}{x}\right)^{2}}-1\right)}\right)- \\
& -4 \alpha \delta \sum\left(\frac{\left(e^{\left(\frac{\delta}{x}\right)^{2}}-1\right)^{-\beta} e^{\left(\frac{\delta}{x}\right)^{2}} \ln \left(e^{\left(\frac{\delta}{x}\right)^{2}}-1\right)}{x^{2}\left(e^{\left(\frac{\delta}{x}\right)^{2}}-1\right)}\right) \\
& L_{233}=L_{323}=L_{332}=4 \sum\left(\frac{e^{\left(\frac{\delta}{x}\right)^{2}}}{x^{2}}\right)+4 \alpha \beta^{2} \delta^{2} \sum\left(\frac{\left(e^{\left(\frac{\delta}{x}\right)^{2}}-1\right)^{-\beta}\left(e^{\left(\frac{\delta}{x}\right)^{2}}\right)^{2} \ln \left(e^{\left(\frac{\delta}{x}\right)^{2}}-1\right)}{x^{4}\left(e^{\left(\frac{\delta}{x}\right)^{2}}-1\right)}\right)- \\
& -8 \alpha \beta \delta^{2} \sum\left(\frac{\left(e^{\left(\frac{\delta}{x}\right)^{2}}-1\right)^{-\beta}\left(e^{\left(\frac{\delta}{x}\right)^{2}}\right)^{2}}{x^{4}\left(e^{\left(\frac{\delta}{x}\right)^{2}}-1\right)^{2}}\right)-2 \alpha \beta \sum\left(\frac{\left(e^{\left(\frac{\delta}{x}\right)^{2}}-1\right)^{-\beta}\left(e^{\left(\frac{\delta}{x}\right)^{2}}\right) \ln \left(e^{\left(\frac{\delta}{x}\right)^{2}}-1\right)}{x^{2}\left(e^{\left(\frac{\delta}{x}\right)^{2}}-1\right)}\right)+ \\
& +2 \alpha \sum\left(\frac{\left(e^{\left(\frac{\delta}{x}\right)^{2}}-1\right)^{-\beta}\left(e^{\left(\frac{\delta}{x}\right)^{2}}\right)^{2}}{x^{2}\left(e^{\left(\frac{\delta}{x}\right)^{2}}-1\right)^{2}}\right)-4 \alpha \beta \delta^{2} \sum\left(\frac{\left(e^{\left(\frac{\delta}{x}\right)^{2}}-1\right)^{-\beta}\left(e^{\left(\frac{\delta}{x}\right)^{2}}\right)^{2} \ln \left(e^{\left(\frac{\delta}{x}\right)^{2}}-1\right)}{x^{4}\left(e^{\left(\frac{\delta}{x}\right)^{2}}-1\right)}\right)+ \\
& +4 \alpha \delta^{2} \sum\left(\frac{\left(e^{\left(\frac{\delta}{x}\right)^{2}}-1\right)^{-\beta}\left(e^{\left(\frac{\delta}{x}\right)^{2}}\right)}{x^{4}\left(e^{\left(\frac{\delta}{x}\right)^{2}}-1\right)}\right)-4 \alpha \delta^{2} \sum\left(\frac{\left(e^{\left(\frac{\delta}{x}\right)^{2}}-1\right)^{-\beta}\left(e^{\left(\frac{\delta}{x}\right)^{2}}\right)^{2}}{x^{4}\left(e^{\left(\frac{\delta}{x}\right)^{2}}-1\right)^{2}}\right)+
\end{aligned}
$$




$$
\begin{aligned}
& +4 \alpha \beta \delta^{2} \sum\left(\frac{\left(e^{\left(\frac{\delta}{x}\right)^{2}}-1\right)^{-\beta}\left(e^{\left(\frac{\delta}{x}\right)^{2}}\right)^{2} \ln \left(e^{\left(\frac{\delta}{x}\right)^{2}}-1\right)}{x^{4}\left(e^{\left(\frac{\delta}{x}\right)^{2}}-1\right)^{2}}\right) \\
& L_{333}=\frac{4}{\delta^{2}}-\delta(\beta+1) \frac{e^{\left(\frac{\delta}{x}\right)^{2}}}{x^{3}}+8 \alpha \beta^{3} \delta^{3} \sum\left(\frac{\left(e^{\left(\frac{\delta}{x}\right)^{2}}-1\right)^{-\beta}\left(e^{\left(\frac{\delta}{x}\right)^{2}}\right)^{3}}{x^{6}\left(e^{\left(\frac{\delta}{x}\right)^{2}}-1\right)^{3}}\right)- \\
& -12 \alpha \beta^{2} \delta^{3} \sum\left(\frac{\left(e^{\left(\frac{\delta}{x}\right)^{2}}-1\right)^{-\beta}\left(e^{\left(\frac{\delta}{x}\right)^{2}}\right)^{2}}{x^{4}\left(e^{\left(\frac{\delta}{x}\right)^{2}}-1\right)^{2}}\right)-24 \alpha \beta^{3} \delta^{3} \sum\left(\frac{\left(e^{\left(\frac{\delta}{x}\right)^{2}}-1\right)^{-\beta}\left(e^{\left(\frac{\delta}{x}\right)^{2}}\right)^{2}}{x^{6}\left(e^{\left(\frac{\delta}{x}\right)^{2}}-1\right)^{2}}\right)+ \\
& +24 \alpha \beta^{2} \delta^{3} \sum\left(\frac{\left(e^{\left(\frac{\delta}{x}\right)^{2}}-1\right)^{-\beta}\left(e^{\left(\frac{\delta}{x}\right)^{2}}\right)^{3}}{x^{6}\left(e^{\left(\frac{\delta}{x}\right)^{2}}-1\right)^{3}}\right)+12 \alpha \beta \delta \sum\left(\frac{\left(e^{\left(\frac{\delta}{x}\right)^{2}}-1\right)^{-\beta}\left(e^{\left(\frac{\delta}{x}\right)^{2}}\right)^{2}}{x^{4}\left(e^{\left(\frac{\delta}{x}\right)^{2}}-1\right)}\right)+ \\
& +12 \alpha \beta \delta \sum\left(\frac{\left(e^{\left(\frac{\delta}{x}\right)^{2}}-1\right)^{-\beta}\left(e^{\left(\frac{\delta}{x}\right)^{2}}\right)}{x^{4}\left(e^{\left(\frac{\delta}{x}\right)^{2}}-1\right)}\right)-12 \alpha \beta \delta \sum\left(\frac{\left(e^{\left(\frac{\delta}{x}\right)^{2}}-1\right)^{-\beta}\left(e^{\left(\frac{\delta}{x}\right)^{2}}\right)^{2}}{x^{4}\left(e^{\left(\frac{\delta}{x}\right)^{2}}-1\right)^{2}}\right)+ \\
& +8 \alpha \beta \delta^{3} \sum\left(\frac{\left(e^{\left(\frac{\delta}{x}\right)^{2}}-1\right)^{-\beta}\left(e^{\left(\frac{\delta}{x}\right)^{2}}\right)}{x^{6}\left(e^{\left(\frac{\delta}{x}\right)^{2}}-1\right)^{2}}\right)-24 \alpha \beta \delta^{3} \sum\left(\frac{\left(e^{\left(\frac{\delta}{x}\right)^{2}}-1\right)^{-\beta}\left(e^{\left(\frac{\delta}{x}\right)^{2}}\right)^{2}}{x^{6}\left(e^{\left(\frac{\delta}{x}\right)^{2}}-1\right)^{2}}\right)+ \\
& +16 \alpha \beta \delta^{3} \sum\left(\frac{\left(e^{\left(\frac{\delta}{x}\right)^{2}}-1\right)^{-\beta}\left(e^{\left(\frac{\delta}{x}\right)^{2}}\right)^{3}}{x^{6}\left(e^{\left(\frac{\delta}{x}\right)^{2}}-1\right)^{3}}\right)
\end{aligned}
$$

If $\phi$ is the parameter to be estimated by an estimator $\hat{\phi}$ then, the square error loss function can be defined as

$$
\phi_{S}(\hat{\phi}, \phi) \propto(\hat{\phi}-\phi)^{2}
$$

This method has been adopted by several authors such as [24] and [25].

[26] defined the Linex loss function as

$$
\phi_{L} \propto q\left(e^{g(\hat{\phi}-\phi)}-g(\hat{\phi}-\phi)-1\right) \quad q>0, g \neq 0
$$

where $\mathrm{q}$ and $\mathrm{g}$ are known as the shape and scale parameters of the loss function. We assume $\mathrm{q}=1$ in this study. According to [27], the Bayes estimator of the linex function is the value $\hat{\phi}$ that minimizes (97) stated as

$$
\hat{\phi}=-\frac{1}{g} \ln \left(E_{\phi}\left[e^{g \phi}\right]\right)
$$

provided that $E_{\phi}\left[e^{c \phi}\right]$ exits. This loss function has been widely used by [28] - [32]

The general Entropy loss function which is also known as modified Linux loss function is defined as

$$
\widehat{\phi}_{E} \propto\left(\frac{\widehat{\phi}}{\phi}\right)^{h}-h \ln \left(\frac{\widehat{\phi}}{\phi}\right)-1
$$


where $\hat{\phi}$ is the parameter estimate of $\phi$. The bayes estimates can be expressed as

$$
\left(E_{\phi}\left[e^{\phi^{-h}}\right]\right)^{-\frac{1}{h}}
$$

provided that $E_{\phi}\left[e^{\phi^{-h}}\right]$ exist. To obtain the various values of the Bayes estimates of the parameters $\alpha, \beta$ and $\delta$. This loss function was adopted by [31] and [32].

4.3.4. Bayes Estimates under the Squared Error Loss Function

1. if $u(\alpha, \beta, \delta)=\hat{\alpha}$

$$
\widehat{\alpha}_{B S}=\hat{\alpha}-\frac{1}{\widehat{\alpha}} \sigma_{11}+\left(\frac{a-1}{\widehat{\beta}}-b\right) \sigma_{12}-\frac{1}{\widehat{\delta}} \sigma_{13}+0.5\left[A \sigma_{11}+B \sigma_{21}+C \sigma_{33}\right]
$$

2. if $u(\alpha, \beta, \delta)=\hat{\beta}$

$$
\hat{\beta}_{B S}=\hat{\beta}-\frac{1}{\widehat{\alpha}} \sigma_{21}+\left(\frac{a-1}{\widehat{\beta}}-b\right) \sigma_{22}-\frac{1}{\hat{\delta}} \sigma_{23}+0.5\left[A \sigma_{12}+B \sigma_{22}+C \sigma_{32}\right]
$$

3. if $u(\alpha, \beta, \delta)=\hat{\delta}$

$$
\hat{\delta}_{B S}=\hat{\delta}-\frac{1}{\widehat{\alpha}} \sigma_{31}+\left(\frac{a-1}{\widehat{\beta}}-b\right) \sigma_{32}-\frac{1}{\widehat{\delta}} \sigma_{33}+0.5\left[A \sigma_{11}+B \sigma_{21}+C \sigma_{33}\right]
$$

4.3.5. The Bayes Estimates under the Linex Loss Function is

1.) if $u(\alpha, \beta, \delta)=e^{g \widehat{\alpha}}$

$$
\hat{\alpha}_{L L}=\hat{\alpha}-\frac{1}{g} \log \left[1-g\left(-\frac{1}{\hat{\alpha}} \sigma_{11}+\left(\frac{a-1}{\widehat{\beta}}-b\right) \sigma_{12}-\frac{1}{\widehat{\delta}} \sigma_{13}-\frac{g}{2} \sigma_{11}+\frac{1}{2}\left[A \sigma_{11}+B \sigma_{21}+C \sigma_{31}\right]\right)\right]
$$

2.) if $u(\alpha, \beta, \delta)=e^{g \widehat{\beta}}$

$$
\hat{\beta}_{L L}=\hat{\beta}-\frac{1}{g} \log \left[1-g\left(-\frac{1}{\hat{\alpha}} \sigma_{21}+\left(\frac{a-1}{\widehat{\beta}}-b\right) \sigma_{22}-\frac{1}{\hat{\delta}} \sigma_{23}-\frac{g}{2} \sigma_{22}+\frac{1}{2}\left[A \sigma_{12}+B \sigma_{22}+C \sigma_{32}\right]\right)\right]
$$

3.) if $u(\alpha, \beta, \delta)=e^{g \widehat{\delta}}$

$$
\hat{\delta}_{L L}=\hat{\delta}-\frac{1}{g} \log \left[1-g\left(-\frac{1}{\hat{\alpha}} \sigma_{31}+\left(\frac{a-1}{\widehat{\beta}}-b\right) \sigma_{32}-\frac{1}{\hat{\delta}} \sigma_{33}-\frac{g}{2} \sigma_{33}+\frac{1}{2}\left[A \sigma_{13}+B \sigma_{23}+C \sigma_{33}\right]\right)\right]
$$

4.3.6. The Bayes Estimates under the General Entropy Loss Function is

1.) $u(\alpha, \beta, \delta)=\hat{\alpha}^{-h}$

$$
\text { if } \hat{\alpha}_{G E}=\left\{\hat{\alpha}^{-h}\left[1-\frac{h}{\widehat{\alpha}}\left(-\frac{1}{\widehat{\alpha}} \sigma_{11}+\left(\frac{a-1}{\widehat{\beta}}-b\right) \sigma_{12}-\frac{1}{\delta} \sigma_{13}-\frac{h+1}{2 \widehat{\alpha}} \sigma_{11}\right)+\frac{1}{2}\left(A \sigma_{11}+B \sigma_{21}+C \sigma_{31}\right)\right]\right\}^{-\frac{1}{h}}
$$

2.) $u(\alpha, \beta, \delta)=\hat{\beta}^{-h}$

$$
\text { if } \hat{\beta}_{G E}=\left\{\hat{\beta}^{-h}\left[1-\frac{h}{\hat{\beta}}\left(-\frac{1}{\hat{\alpha}} \sigma_{21}+\left(\frac{a-1}{\widehat{\beta}}-b\right) \sigma_{22}-\frac{1}{\delta} \sigma_{23}-\frac{h+1}{2 \widehat{\beta}} \sigma_{22}\right)+\frac{1}{2}\left(A \sigma_{12}+B \sigma_{22}+C \sigma_{32}\right)\right]\right\}^{-\frac{1}{h}}
$$

3.) $u(\alpha, \beta, \delta)=\hat{\delta}^{-h}$

$$
\text { if } \hat{\delta}_{G E}=\left\{\hat{\delta}^{-h}\left[1-\frac{h}{\hat{\delta}}\left(-\frac{1}{\hat{\alpha}} \sigma_{31}+\left(\frac{a-1}{\widehat{\beta}}-b\right) \sigma_{32}-\frac{1}{\delta} \sigma_{33}-\frac{h+1}{2 \widehat{\delta}} \sigma_{33}\right)+\frac{1}{2}\left(A \sigma_{13}+B \sigma_{23}+C \sigma_{33}\right)\right]\right\}^{-\frac{1}{h}}
$$

\subsection{Simulation Study}

In this section, we generate 2000 random samples $X_{1}, \ldots, X_{\mathrm{n}}$ of sizes $n=25,60,100$ and 500 from the new Weibull Inverse Rayleigh distribution via the quantile function (inverse transformation method of simulation) at different parameter values for $\alpha, \beta$, and $\delta$. We considered the following set of values for the parameter combinations $(\alpha, \beta, \delta)$ as follows: $(\alpha, \beta, \delta)=[(0.5,0.5,0.5),(1,1.5,1)$ and $(0.7,1,0.4)], \mathrm{g}=(6,7,10), \mathrm{h}=(7,8)$ and $(\mathrm{a}, \mathrm{b})=[(1.2,0.3),(2$, 2), (2,1.5)].

The results presented in Table 2 displayed the true values set for the three parameters $(\alpha, \beta, \delta)$ of the new WIR distribution, their estimated values as well as their standard errors in brackets. Thus, the four estimators of these parameters $(\alpha, \beta, \delta)$ as employed in these works are the Maximum Likelihood Estimator (ML) $\hat{\alpha}_{M L}, \hat{\beta}_{M L}, \hat{\delta}_{M L}$, the 
Bayesian Estimators under three loss functions( i.) the Square Error Loss Function (BS) $\hat{\alpha}_{B S}, \hat{\beta}_{B S}, \hat{\delta}_{B S}$ (ii.) the Linex Loss Function (LL) $\hat{\alpha}_{L L}, \hat{\beta}_{L L}, \hat{\delta}_{L L}$ and (iii.) the General Entropy Loss function (GE) $\hat{\alpha}_{G E}, \hat{\beta}_{G E}, \hat{\delta}_{G E}$

Table 2. Average estimates of the parameters of the new WIR distributions at $\alpha=0.5, \beta=0.5$ and $\delta=0.5$ when $\mathrm{g}=7, \mathrm{~h}=7, \mathrm{a}=1.2, \mathrm{~b}=0.3$. The MSEs of the parameter estimates are in parentheses

\begin{tabular}{|c|c|c|c|c|}
\hline Sample Size & Method & $\alpha=0.5$ & $\beta=0.5$ & $\delta=0.5$ \\
\hline \multirow{4}{*}{25} & ML & $\begin{array}{c}0.3072 \\
(0.2411)\end{array}$ & $\begin{array}{c}0.6767 \\
(0.1418)\end{array}$ & $\begin{array}{c}0.4411 \\
(0.1494)\end{array}$ \\
\hline & BS & $\begin{array}{c}0.3165 \\
(0.0337)\end{array}$ & $\begin{array}{c}0.6907 \\
(0.0364)\end{array}$ & $\begin{array}{l}0.4747 \\
(0.006)\end{array}$ \\
\hline & LL & $\begin{array}{c}0.2992 \\
(0.0403)\end{array}$ & $\begin{array}{c}0.6613 \\
(0.0260)\end{array}$ & $\begin{array}{c}0.4470 \\
(0.0028)\end{array}$ \\
\hline & GE & $\begin{array}{c}0.2754 \\
(0.0504) \\
\end{array}$ & $\begin{array}{c}0.6513 \\
(0.0229) \\
\end{array}$ & $\begin{array}{c}0.4460 \\
(0.0229) \\
\end{array}$ \\
\hline \multirow{4}{*}{60} & ML & $\begin{array}{c}0.5012 \\
(0.1659)\end{array}$ & $\begin{array}{c}0.4747 \\
(0.0613)\end{array}$ & $\begin{array}{c}0.4932 \\
(0.0858)\end{array}$ \\
\hline & BS & $\begin{array}{c}0.4285 \\
(0.0051)\end{array}$ & $\begin{array}{l}0.40746 \\
(0.0051)\end{array}$ & $\begin{array}{c}0.4358 \\
(0.0041)\end{array}$ \\
\hline & LL & $\begin{array}{l}0.4065 \\
(0.009)\end{array}$ & $\begin{array}{c}0.4139 \\
(0.0074)\end{array}$ & $\begin{array}{c}0.5093 \\
(0.0027)\end{array}$ \\
\hline & GE & $\begin{array}{c}0.4448 \\
(0.0030)\end{array}$ & $\begin{array}{c}0.4407 \\
(0.0035)\end{array}$ & $\begin{array}{c}0.5036 \\
(0.0035)\end{array}$ \\
\hline \multirow{4}{*}{100} & ML & $\begin{array}{c}0.4550 \\
(0.1233)\end{array}$ & $\begin{array}{c}0.4849 \\
(0.0480)\end{array}$ & $\begin{array}{l}0.4524 \\
(0.064)\end{array}$ \\
\hline & BS & $\begin{array}{c}0.4207 \\
(0.0043)\end{array}$ & $\begin{array}{l}0.4538 \\
(0.002)\end{array}$ & $\begin{array}{c}0.4265 \\
(0.0030)\end{array}$ \\
\hline & LL & $\begin{array}{l}0.4049 \\
(0.009)\end{array}$ & $\begin{array}{c}0.4530 \\
(0.0022)\end{array}$ & $\begin{array}{c}0.4602 \\
(0.0020)\end{array}$ \\
\hline & GE & $\begin{array}{c}0.4235 \\
(0.0028)\end{array}$ & $\begin{array}{c}0.4655 \\
(0.0012)\end{array}$ & $\begin{array}{c}0.4572 \\
(0.0012)\end{array}$ \\
\hline \multirow{4}{*}{500} & ML & $\begin{array}{c}0.4894 \\
(0.1121)\end{array}$ & $\begin{array}{c}0.5089 \\
(0.0425)\end{array}$ & $\begin{array}{c}0.4824 \\
(0.0570)\end{array}$ \\
\hline & BS & $\begin{array}{c}0.4560 \\
(0.0019)\end{array}$ & $\begin{array}{c}0.4779 \\
(0.0005)\end{array}$ & $\begin{array}{c}0.4552 \\
(0.0020)\end{array}$ \\
\hline & LL & $\begin{array}{c}0.4445 \\
(0.0031)\end{array}$ & $\begin{array}{c}0.4798 \\
(0.0004)\end{array}$ & $\begin{array}{c}0.4890 \\
(0.0001)\end{array}$ \\
\hline & GE & $\begin{array}{c}0.4610 \\
(0.0015)\end{array}$ & $\begin{array}{c}0.4912 \\
(0.00008)\end{array}$ & $\begin{array}{c}0.4863 \\
(0.00008)\end{array}$ \\
\hline
\end{tabular}


Table 3. Average estimates of the parameters of the new WIR distributions at $\boldsymbol{\alpha}=1, \boldsymbol{\beta}=\mathbf{1 . 5}$ and $\boldsymbol{\delta}=\mathbf{1}$ when $\mathrm{g}=6$, h=8, a=2, b=2. The MSEs of the parameter estimates are in parentheses

\begin{tabular}{|c|c|c|c|c|}
\hline Sample Size & Method & $\alpha=1$ & $\beta=1.5$ & $\delta=1$ \\
\hline \multirow{4}{*}{25} & ML & $\begin{array}{c}0.8964 \\
(3.2355)\end{array}$ & $\begin{array}{c}1.9790 \\
(0.9044)\end{array}$ & $\begin{array}{c}0.9702 \\
(0.6244)\end{array}$ \\
\hline & BS & $\begin{array}{c}0.7166 \\
(0.0803)\end{array}$ & $\begin{array}{c}1.7789 \\
(0.0778)\end{array}$ & $\begin{array}{c}1.0103 \\
(0.0001)\end{array}$ \\
\hline & LL & $\begin{array}{c}0.7480 \\
(0.0635)\end{array}$ & $\begin{array}{c}1.7818 \\
(0.0794)\end{array}$ & $\begin{array}{c}0.9877 \\
(0.0001)\end{array}$ \\
\hline & GE & $\begin{array}{c}0.7607 \\
(0.0572)\end{array}$ & $\begin{array}{c}1.7910 \\
(0.0847)\end{array}$ & $\begin{array}{c}0.9882 \\
(0.0847)\end{array}$ \\
\hline \multirow{4}{*}{60} & ML & $\begin{array}{c}1.3387 \\
(1.5272)\end{array}$ & $\begin{array}{c}1.3357 \\
(0.3354)\end{array}$ & $\begin{array}{c}1.0808 \\
(0.3205)\end{array}$ \\
\hline & BS & $\begin{array}{c}1.0435 \\
(0.0018)\end{array}$ & $\begin{array}{c}1.1621 \\
(0.1142)\end{array}$ & $\begin{array}{c}1.1029 \\
(0.0106)\end{array}$ \\
\hline & LL & $\begin{array}{c}1.1310 \\
(0.0171)\end{array}$ & $\begin{array}{c}1.2200 \\
(0.0784)\end{array}$ & $\begin{array}{c}1.0935 \\
(0.0087)\end{array}$ \\
\hline & GE & $\begin{array}{c}1.1434 \\
(0.0206)\end{array}$ & $\begin{array}{c}1.2220 \\
(0.0773)\end{array}$ & $\begin{array}{c}1.0933 \\
(0.0773)\end{array}$ \\
\hline \multirow{4}{*}{100} & ML & $\begin{array}{c}0.6647 \\
(0.7451)\end{array}$ & $\begin{array}{c}1.4802 \\
(0.2576)\end{array}$ & $\begin{array}{c}0.9021 \\
(0.2367)\end{array}$ \\
\hline & BS & $\begin{array}{c}0.6493 \\
(0.1230)\end{array}$ & $\begin{array}{c}1.4602 \\
(0.0016)\end{array}$ & $\begin{array}{c}0.9146 \\
(0.0073)\end{array}$ \\
\hline & $\mathrm{LL}$ & $\begin{array}{c}0.6402 \\
(0.1295)\end{array}$ & $\begin{array}{c}1.4399 \\
(0.0036)\end{array}$ & $\begin{array}{c}0.9057 \\
(0.0089)\end{array}$ \\
\hline & $\mathrm{GE}$ & $\begin{array}{c}0.6286 \\
(0.1379)\end{array}$ & $\begin{array}{c}1.4405 \\
(0.0035)\end{array}$ & $\begin{array}{c}0.9057 \\
(0.0035)\end{array}$ \\
\hline \multirow{4}{*}{500} & ML & $\begin{array}{c}1.0671 \\
(0.9644)\end{array}$ & $\begin{array}{c}1.4938 \\
(0.2400)\end{array}$ & $\begin{array}{c}1.0059 \\
(0.2118)\end{array}$ \\
\hline & BS & $\begin{array}{c}1.0259 \\
(0.0007)\end{array}$ & $\begin{array}{c}1.4631 \\
(0.0014)\end{array}$ & $\begin{array}{c}1.0159 \\
(0.0003)\end{array}$ \\
\hline & LL & $\begin{array}{c}1.0175 \\
(0.0003)\end{array}$ & $\begin{array}{c}1.4515 \\
(0.0024)\end{array}$ & $\begin{array}{c}1.0095 \\
(0.00009)\end{array}$ \\
\hline & GE & $\begin{array}{c}1.0130 \\
(0.0002)\end{array}$ & $\begin{array}{c}1.4526 \\
(0.0022)\end{array}$ & $\begin{array}{c}1.0095 \\
(0.0023) \\
\end{array}$ \\
\hline
\end{tabular}


Table 4. Average estimates of the parameters of the new WIR distributions at $\alpha=0.7, \beta=1$ and $\delta=0.4$ when $\mathrm{g}=10$, $\mathrm{h}=8$, a $=2$, $\mathrm{b}=1.5$. The MSEs of the parameter estimates are in parentheses

\begin{tabular}{|c|c|c|c|c|}
\hline Sample Size & Method & $\alpha=0.7$ & $\beta=1$ & $\delta=0.4$ \\
\hline \multirow{4}{*}{25} & ML & $\begin{array}{c}0.2853 \\
(0.6977)\end{array}$ & $\begin{array}{c}1.4209 \\
(0.4415)\end{array}$ & $\begin{array}{c}0.3285 \\
(0.1975)\end{array}$ \\
\hline & $\mathrm{BS}$ & $\begin{array}{c}0.2775 \\
(0.1785)\end{array}$ & $\begin{array}{c}1.3915 \\
(0.1533)\end{array}$ & $\begin{array}{c}0.3459 \\
(0.0029)\end{array}$ \\
\hline & $\mathrm{LL}$ & $\begin{array}{c}0.2620 \\
(0.1918)\end{array}$ & $\begin{array}{c}1.3409 \\
(0.1162)\end{array}$ & $\begin{array}{c}0.3318 \\
(0.0046)\end{array}$ \\
\hline & GE & $\begin{array}{c}0.2506 \\
(0.2019)\end{array}$ & $\begin{array}{c}1.3568 \\
(0.1273) \\
\end{array}$ & $\begin{array}{c}0.3308 \\
(0.1273) \\
\end{array}$ \\
\hline \multirow{4}{*}{60} & ML & $\begin{array}{c}0.7745 \\
(0.5124)\end{array}$ & $\begin{array}{c}0.9226 \\
(0.165\end{array}$ & $\begin{array}{l}0.4139 \\
(0.962)\end{array}$ \\
\hline & $\mathrm{BS}$ & $\begin{array}{c}0.7623 \\
(0.0039)\end{array}$ & $\begin{array}{c}0.9200 \\
(0.0079)\end{array}$ & $\begin{array}{c}0.4257 \\
(0.0007)\end{array}$ \\
\hline & $\mathrm{LL}$ & $\begin{array}{c}0.7307 \\
(0.0009)\end{array}$ & $\begin{array}{c}0.8846 \\
(0.0133)\end{array}$ & $\begin{array}{c}0.4157 \\
(0.0002)\end{array}$ \\
\hline & GE & $\begin{array}{c}0.7273 \\
(0.0007)\end{array}$ & $\begin{array}{c}0.8858 \\
(0.0130)\end{array}$ & $\begin{array}{l}0.4152 \\
(0.013)\end{array}$ \\
\hline \multirow{4}{*}{100} & ML & $\begin{array}{c}0.5538 \\
(0.3250)\end{array}$ & $\begin{array}{c}0.9776 \\
(0.1281)\end{array}$ & $\begin{array}{c}0.3628 \\
(0.0716)\end{array}$ \\
\hline & BS & $\begin{array}{c}0.5492 \\
(0.0227)\end{array}$ & $\begin{array}{c}0.9726 \\
(0.0008)\end{array}$ & $\begin{array}{c}0.3682 \\
(0.0018)\end{array}$ \\
\hline & $\mathrm{LL}$ & $\begin{array}{c}0.5372 \\
(0.0265)\end{array}$ & $\begin{array}{c}0.9564 \\
(0.0019)\end{array}$ & $\begin{array}{c}0.3638 \\
(0.0640)\end{array}$ \\
\hline & GE & $\begin{array}{c}0.5304 \\
(0.0288) \\
\end{array}$ & $\begin{array}{c}0.9579 \\
(0.0018)\end{array}$ & $\begin{array}{c}0.3635 \\
(0.0018)\end{array}$ \\
\hline \multirow{4}{*}{500} & ML & $\begin{array}{c}0.7004 \\
(0.3466)\end{array}$ & $\begin{array}{c}1.0081 \\
(0.1172)\end{array}$ & $\begin{array}{c}0.3948 \\
(0.0640)\end{array}$ \\
\hline & $\mathrm{BS}$ & $\begin{array}{c}0.6955 \\
(0.00002)\end{array}$ & $\begin{array}{c}1.0030 \\
(0.000009)\end{array}$ & $\begin{array}{c}0.3991 \\
(0.0000009)\end{array}$ \\
\hline & $\mathrm{LL}$ & $\begin{array}{c}0.6830 \\
(0.0003)\end{array}$ & $\begin{array}{c}0.9897 \\
(0.0001)\end{array}$ & $\begin{array}{c}0.3955 \\
(0.00002)\end{array}$ \\
\hline & GE & $\begin{array}{c}0.6794 \\
(0.0004) \\
\end{array}$ & $\begin{array}{c}0.9914 \\
(0.00007) \\
\end{array}$ & $\begin{array}{c}0.3953 \\
(0.00007) \\
\end{array}$ \\
\hline
\end{tabular}

The results of the simulation exercises are presented in Tables 2 to 4 . It can be observed from the various results in the tables that the estimated values for all three parameters are close to their true values. Expectedly, the mean squares errors of the parameters decrease as sample sizes increase for all the estimates provided by the four estimators (ML, BS, LL, and GE). 

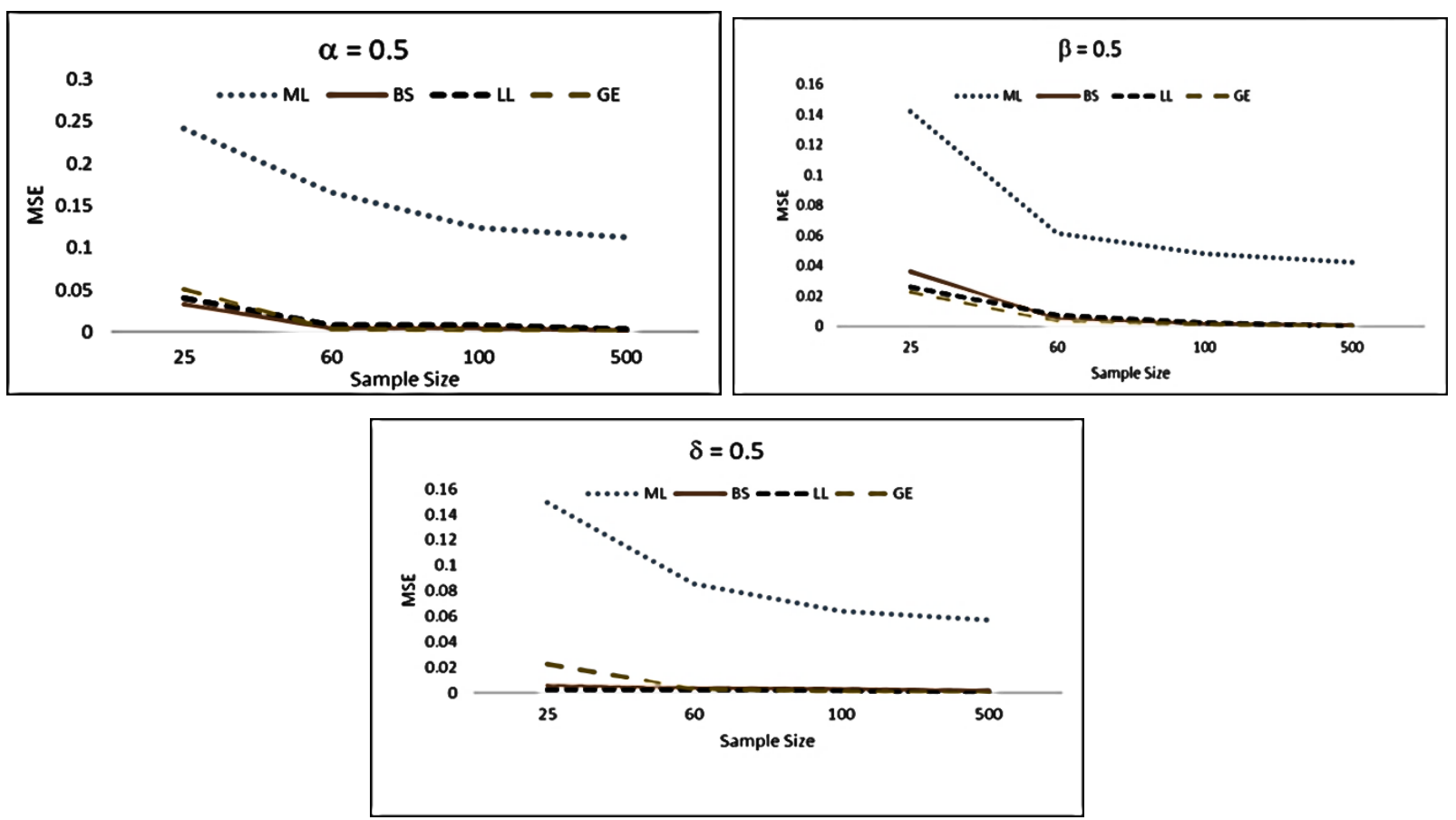

Figure 3. Graphical presentations of the performances of ML, BS, LL, and GE estimators of WIR distribution when $\alpha=0.5, \beta=0.5$ and $\delta=0.5$ at different sample sizes

We observed that the results obtained under both the Bayesian and Classical methods become better as the sample size increases. Also, we deduced that as the sample sizes increase the estimated values for the three parameters approached the true value of the parameters. In terms of MSE, the Bayesian estimators with relatively smaller MSEs under the three-loss functions provided better results than the MLE at all the sample sizes considered. However, within the Bayesian context, all the Bayesian estimators under the three-loss functions considered compete favorably with one another, especially at sample sizes above 25 .

\section{Applications}

In this section, we demonstrate the applications of the new WIR distribution on three real-life datasets. The first two datasets are the cases of the COVID-19 dataset (January 21 to March 27, 2020) as reported by WHO (2020) and Worldometers (2020). The third data were on the Strength measured in GPA for single carbon fibers and impregnated 1000 -carbon fiber tows at gauge lengths of $20 \mathrm{~mm}$ as reported in [33].

Data 1: Daily Cases of (COVID-19) across the world between January 21 to March 27, 2020 (WHO, 2020).

60, 32, 265, 472, 698, 785, 1781, 1477, 1755, 2010, 2127, 2603, 2838, 3239, 3915, 3721, 3173 3437, 2676, 3001, 2546, 2035, 14153, 5151, 2662, 2097, 2132, 2003, 1852, 516, 977, 996, 978 554, 882, 741, 992, 1292, 1503, 1989, 1981, $1858,2573,2298,3111,3625,4049,3892,4390,4567,7266,8295,10907,11059,13042,12897,15745,20585,26158$, $30648,29429,32480,41371,43744,48461,60830,64501$

Table 5. Summary Statistics of Daily Cases of COVID-19 data

\begin{tabular}{ccccccccc}
\hline Min. & 1st Quarter & Median & Mean & 3rd quarter & Max & $\begin{array}{c}\text { Standard } \\
\text { deviation }\end{array}$ & Kurtosis & Skewness \\
\hline 32 & 1,629 & 2,662 & 8,894 & 7,780 & 64,501 & $14,538.08$ & 7.858 & 2.352 \\
\hline
\end{tabular}

Tables 5 shows that the daily cases of COVID-19 are positively skewed with high kurtosis (leptokurtic) which means it has heavier tails than the normal distribution. 
Table 6. Summary of the Maximum Likelihood Estimates and the Standard Errors of the new WIR distribution for Daily Cases of the COVID-19 data set.

\begin{tabular}{ccccccc}
\hline & ML Estimates & Std. Errors & t-values & $\begin{array}{c}\text { SELF } \\
\text { Estimates }\end{array}$ & $\begin{array}{c}\text { Linex } \\
\text { Estimates }\end{array}$ & $\begin{array}{c}\text { General Entropy } \\
\text { Estimates }\end{array}$ \\
\hline A & 0.04298 & 0.01496 & 2.873 & 1.7142 & 0.0495 & 0.0406 \\
\hline B & 0.34508 & 0.03104 & 11.117 & 2.0169 & 0.3563 & 0.3390 \\
\hline$\Delta$ & 69.56424 & 0.09388 & 740.956 & 7.9792 & 69.3414 & 69.3734 \\
\hline
\end{tabular}

Table 6 shows the maximum likelihood estimates of the model parameters on the COVID-19 dataset and their corresponding standard errors.

Table 7. Goodness of fit of WIR distribution for Daily Cases of COVID 19

\begin{tabular}{cccccc}
\hline Log-Likelihood: & AIC & BIC & HQIC & KS Statistic & K-S p-value \\
\hline-665.5231 & 1337.046 & 1343.66 & 1339.663 & 0.089552 & 0.5843 \\
\hline
\end{tabular}

The estimate of the log-likelihood is -665.5231 , the value of the models Kolmogorov-Smirnov (K-S) statistic is 0.089552 with a corresponding p-value of 0.5843 , which shows that the new proposed distribution (WIR) fit the COVID-19 dataset at any reasonable significance level.

Data 2: Daily Death of COVID-19 across the world between January 21 to March 27, 2020 (Worldometers, 2020).

$3,3,8,16,15,24,26,26,38,43,46,45,58,64,66,73,73,86,89,97,108,97,146,122,143,143,106,98,136,117$, $121,113,100,158,81,64,37,58,65,54,73,67,85,83,102,107,105,228,198,271,332,353,447,405,687,642$, $817,972,1079,1356,1625,1629,1873,2381,2388,2791,3271$.

Table 8. Summary Statistics for Daily Death of COVID 19

\begin{tabular}{cccccccc}
\hline Min. & 1st Quarter & Median & Mean & 3rd Quarter & Max & Kurtosis & Skewness \\
\hline 3 & 64 & 102 & 408 & 301.5 & 3271 & 8.0985 & 2.4161 \\
\hline
\end{tabular}

Tables 8 shows that the daily death of COVID 19 is also positively skewed with high kurtosis which means it has heavier tails than the normal distribution

Table 9. Summary of WIR distribution MLE and Standard Error of Daily Death of COVID 19

\begin{tabular}{ccccccc}
\hline & $\begin{array}{c}\text { ML } \\
\text { Estimates }\end{array}$ & Std. Error & t-test & $\begin{array}{c}\text { SELF } \\
\text { Estimates }\end{array}$ & $\begin{array}{c}\text { Linex } \\
\text { Estimates }\end{array}$ & $\begin{array}{c}\text { General Entropy } \\
\text { Estimates }\end{array}$ \\
\hline A & 0.09092 & 0.02606 & 3.489 & 0.2663 & 0.0924 & 0.0870 \\
\hline B & 0.32143 & 0.02878 & 11.17 & 0.4991 & 0.3230 & 0.3203 \\
\hline$\Delta$ & 6.65036 & 0.23947 & 27.771 & 6.9871 & 6.7934 & 6.5747 \\
\hline
\end{tabular}

Table 10. Goodness of fit of WIR distribution for Daily Cases of COVID 19

\begin{tabular}{cccccc}
\hline Log-Likelihood: & AIC & BIC & HQIC & K-S Statistic & K-S P-value \\
\hline-453.9026 & 913.8051 & 920.4192 & 916.4223 & 0.074627 & 0.6886 \\
\hline
\end{tabular}

The estimate of the log-likelihood is -453.9026 , the value of the models Kolmogorov-Smirnov (K-S) statistic is 0.074627 with a corresponding p-value of 0.6886 , which shows that the new proposed distribution (WIR) fits Daily Cases of COVID-19 data very well. It can be noticed from Table 10 that the K-S p-value of the WIR model is very closed to 1 .

Data 3: carbon fibers Strength $(20 \mathrm{~mm})$ Data set

$1.312,1.314,1.479,1.552,1.700,1.803,1.861,1.865,1.944,1.958,1.966,1.997,2.006,2.021,2.027,2.055,2.063$, $2.098,2.14,2.179,2.224,2.240,2.253,2.270,2.272,2.274,2.301,2.301,2.359,2.382,2.382,2.426,2.434,2.435$, $2.478,2.490,2.511,2.514,2.535,2.554,2.566,2.57,2.586,2.629,2.633,2.642,2.648,2.684,2.697,2.726,2.770$, $2.773,2.800,2.809,2.818,2.821,2.848,2.88,2.954,3.012,3.067,3.084,3.090,3.096,3.128,3.233,3.433,3.585$, 3.585 .

The strength data was originally reported by [33] where the strength is measured in GPA for single carbon fibers and impregnated 1000-carbon fiber tows at gauge lengths of $20 \mathrm{~mm}$. These data set were fitted to the Half- Logistics Inverse Rayleigh (HLIR) distribution by [10] and in [11] the Type II Topp-Leone Inverse Rayleigh (T2TLIR) distribution was 
fitted to the data. Other distributions that have been fitted to these sam data are the Transmuted Inverse Rayleigh distribution (TIR) [12], the Odd Frechet Inverse Rayleigh (OFIR) distribution [13] and one parameter Inverse Rayleigh (IR) distribution [13].

The Akaike Information Criterion (AIC), Bayesian information criterion (BIC), and Hannan-Quinn information criterion (HQIC) are used to compare the performances of all the models on the three data sets employed.

Table 11. The MLEs, SEs (parentheses) and - log likelihood of the new WIR distribution and those of the other five existing related distributions on the Strength (20mm) data set (data 3 )

\begin{tabular}{|c|c|c|c|c|}
\hline Models & & MLE(SE) & & -log likelihood \\
\hline $\mathrm{WIR}(\alpha, \beta, \delta)$ & $\begin{array}{c}0.3232 \\
(0.9469)\end{array}$ & $\begin{array}{c}2.1396 \\
(0.5469)\end{array}$ & $\begin{array}{c}1.7896 \\
(0.8746)\end{array}$ & 48.8915 \\
\hline $\operatorname{HLIR}(\alpha, \lambda)$ & $\begin{array}{c}3.6538 \\
(0.2197)\end{array}$ & $\begin{array}{l}10.2773 \\
(2.5587)\end{array}$ & - & 50.5018 \\
\hline $\operatorname{T} 2 \operatorname{TLIR}(\alpha, \theta)$ & $\begin{array}{c}2.7966 \\
(0.1574)\end{array}$ & $\begin{array}{l}10.2992 \\
(2.8538)\end{array}$ & - & 52.0685 \\
\hline $\operatorname{TIR}(\theta, \lambda)$ & $\begin{array}{c}7.5093 \\
(19.4108)\end{array}$ & $\begin{array}{c}0.8891 \\
(0.0182)\end{array}$ & - & 71.9390 \\
\hline $\operatorname{OFIR}(\theta, \alpha)$ & $\begin{array}{c}2.9540 \\
(0.1862)\end{array}$ & $\begin{array}{c}1.3910 \\
(0.1231)\end{array}$ & - & 71.7113 \\
\hline $\operatorname{IR}(\alpha)$ & $\begin{array}{c}2.2827 \\
(0.1374)\end{array}$ & - & - & 88.4130 \\
\hline
\end{tabular}

Table 12. Goodness-of-fit measures based on AIC, BIC, HQIC, K-S values and their corresponding ranks (in parentheses) for the Strength (20mm) data set (data 3)

\begin{tabular}{|c|c|c|c|c|c|}
\hline Models & AIC & BIC & HQIC & K-S Value & P-value \\
\hline $\operatorname{WIR}(\alpha, \beta, \delta)$ & $103.7831(1)$ & 110.4854 (2) & $106.4421(1)$ & 0.029 & 0.9437 \\
\hline $\operatorname{HLIR}(\alpha, \lambda)$ & $105.0030(2)$ & $109.4720(1)$ & $106.7764(2)$ & 0.0596 & 0.9668 \\
\hline $\operatorname{T2TLIR}(\alpha, \theta)$ & $108.1371(3)$ & $112.6053(3)$ & $109.9098(3)$ & 0.0776 & 0.7993 \\
\hline $\operatorname{TIR}(\theta, \lambda)$ & 145.8787 (4) & 148.1128 & $146.7651(4)$ & 0.254 & 0.0002 \\
\hline $\operatorname{OFIR}(\theta, \alpha)$ & $147.4228(5)$ & $151.8910(5)$ & $149.1955(5)$ & 0.1801 & 0.0227 \\
\hline $\operatorname{IR}(\alpha)$ & $178.8262(6)$ & $181.0603(6)$ & $179.7125(6)$ & 0.3549 & 0.0000 \\
\hline
\end{tabular}

Table 11 shows the summary statistics of the WIR, HLIR, TIR, OFIR, and IR distributions. These five distributions are fitted to the data 3 using maximum likelihood estimation. The MLES of the parameters (with their standard errors) are discussed in Table 9 and their corresponding log-likelihood values. AIC, BIC, HQIC and p-values are displayed in Table 10. Hence, the new WIR model provides the best fit among the other models for data3 as shown in Table 12, since it has the lowest values of AIC, BIC, and K-S Values. 


\section{COVID-19 Data set}

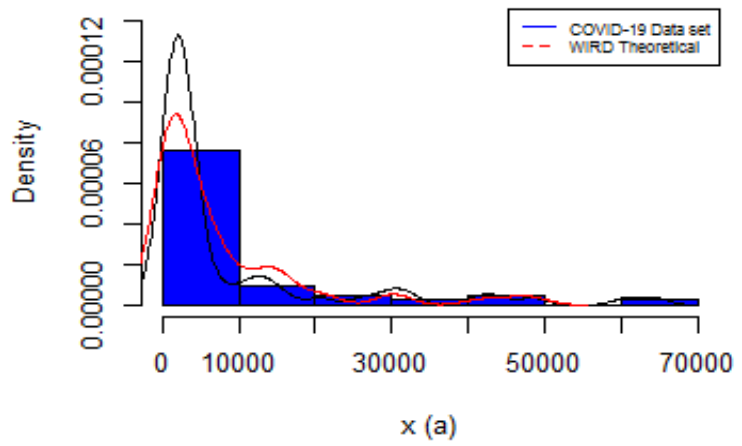

\section{COVID-19 Data set}

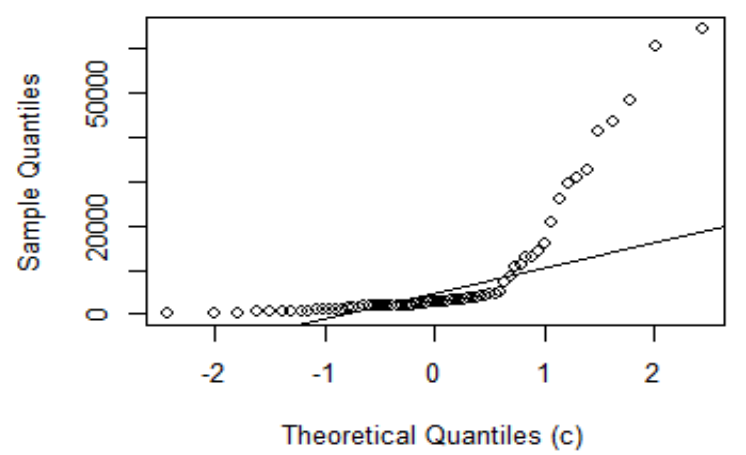

COVID-19 Data set
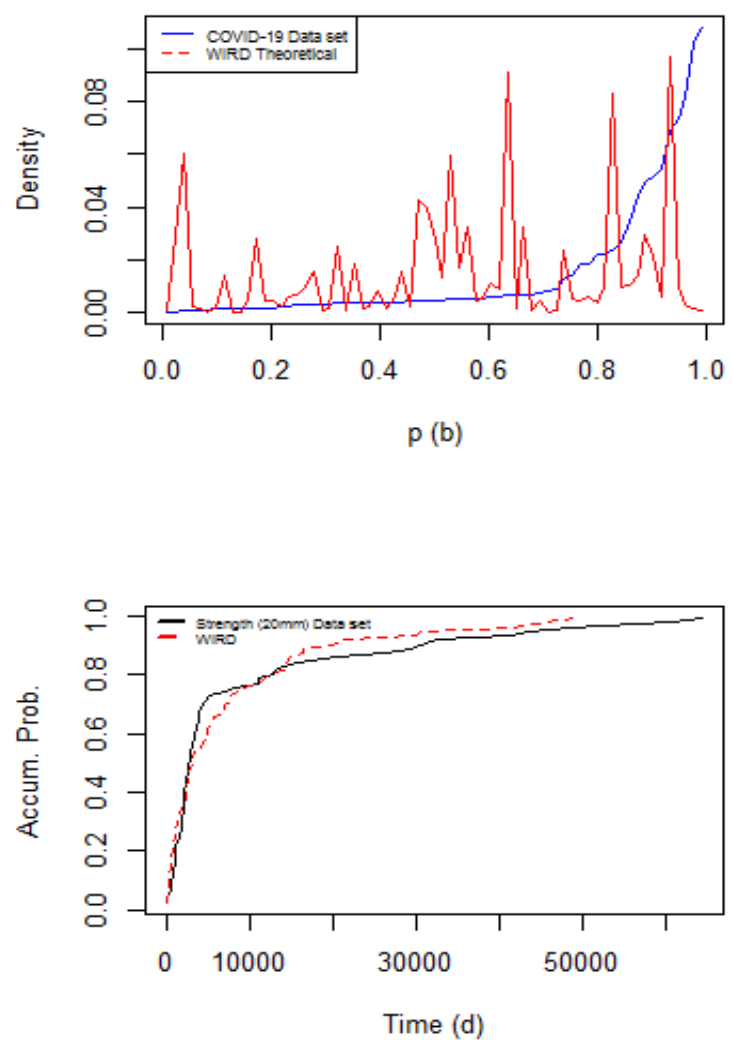

Figure 4. (a) Histogram and Empirical density of COVID 19 data with WIR distribution superimposed, (b) Probability plot and time plot. (c) The Quantiles distribution and (d) the empirical CDF of COVID- 19 and theoretical CDF of the WIR distribution on a dataset

Figure 4 (a-c), displayed the histogram and empirical density, probability plot and time plot, quantiles distribution, empirical CDF, and theoretical CDF for the WIR distribution on the COVID- 19 dataset.
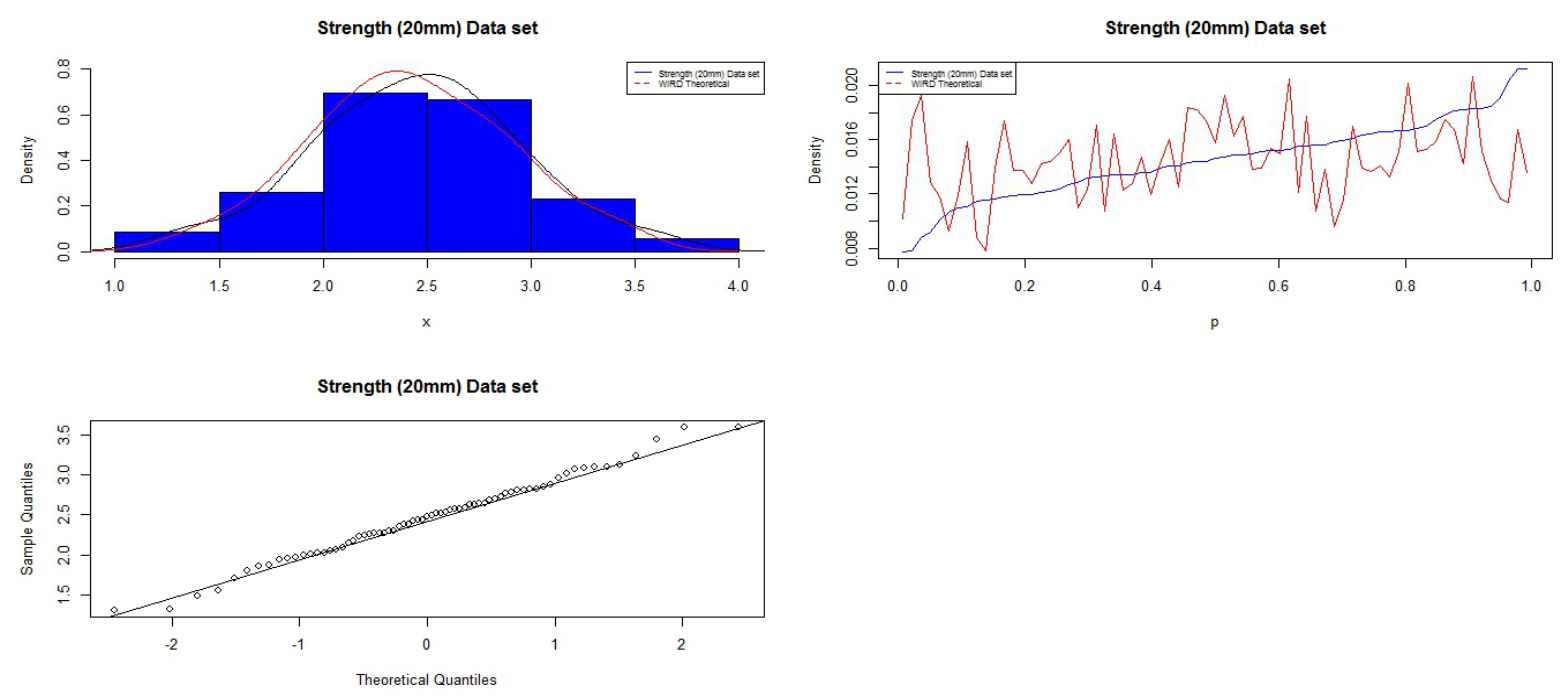

Figure 5. (a) Histogram and Empirical density of Carbon fibers strength data with WIR distribution superimposed, (b) Probability plot and time plot. (c) The Quantiles distribution of the data set against Qunatiles distribution of fitted model WIR distribution on the Strength (20mm) data set (data 3)

Figure 5 (a-c) fitted PDF, time plot and Q-Q plots of the new WIR distribution for the Strength (20mm) data set (data 3) are displayed in Fig 5. 


\section{Conclusions}

In this research paper, we propose a new three-parameters WIR distribution. Some mathematical properties of the new WIR distribution such as moment, order statistic, asymptotic, skewness, kurtosis, and its monotonic likelihood property were investigated. The method of the maximum likelihood estimation and Bayesian method were adopted to estimate the parameters of the distribution. Figures 1 shows the density plot for different scale and shape parameter values. Figure 2 displayed the hazard function for different values of scale and shape parameters. Figure 3, shows the MSEs for the four types of estimations (ML, BS, LL, and GE) adopted in this study. It can be seen that the MSEs for the Bayesian estimators (BS, LL, and GE) are smaller than that of the classical method (ML). Also, as the sample size increases the MSEs decrease for all four estimations. Figure $4(a-c)$, displayed the histogram and empirical density, probability plot and time plot, quantiles distribution, empirical CDF, and theoretical CDF for the WIR distribution on the COVID- 19 dataset. Figure 5 (a-c) fitted PDF, time plot, and Q-Q plots of the new WIR distribution for the Strength (20mm) data set (data 3).

The new WIR distribution was fitted to three real-life data sets to demonstrate its application and efficiency. All the results obtained showed that the proposed distribution provided the best fit compared to other competing models of the same baseline distributions based on several models' selection criteria adopted in this work.

Further results from simulation studies showed that the estimated values of all three parameters of the new WIR distribution are close to their true values. Consequently, it was observed that the standard errors of the parameters are relatively smaller than those provided by other competing existing distributions compared and that these standard errors reduce as the sample sizes increase for all the parameter values specified in the simulation study.

Fundamentally, the results from this work showed that the Bayesian estimates of the new WIR distribution are better than those provided by the classical frequentist method of MLE as can be observed from Table 2 to 4 and graphically displayed by Fig 3 . The MSEs under the Bayesian methods are less than that of the classical approach.

Although, the performances of both the MLE and Bayesian estimators of the new WIR distribution are not worst off as can be observed in various results, the superiority of the Bayesian estimators over the MLE necessitates our recommendation here that the Bayesian estimation techniques be employed in future applications of the new WIR distribution for better efficiency irrespective of the sample sizes.

\section{REFERENCES}

[1] Tahir, M. H., Zubair, M., Cordeiro, G. M., Alzaatreh, A., and Mansoor, M. The Weibull-power Cauchy distribution: Model, properties and applications. Hacettepe Journal of Mathematics and Statistics, 2017. 46(4). https://doi.org/10. 15672/HJMS.20159814103.

[2] Aljarrah, M.A., Lee, C., Famoye, F. On generating T-X family of distributions using quantile functions, Journal of Statistical Distributions and Applications, 2014, 1(2), doi:10.1890/13-1452.1.

[3] Alzaatreh, A., Lee, C. and Famoye, F. T-normal family of distributions: a new approach to generalize the normal distribution. Journal of Statistical Distributions and Applications, 2014, 1:16.

[4] Alzaatreh, A., Lee, C., Famoye, F., Ghosh, I. The generalized Cauchy family of distributions with applications. Journal of Statistical Distributions and Applications, 2016, 3(12), 1-16.

[5] Alzaatreh, A., Lee, C., and Famoye, F. Family of generalized gamma distributions: Properties and applications. Hacettepe Journal of Mathematics and Statistics, 2016, 45(3), 869-886. https://doi.org/10.15672/HJMS.20156610980.

[6] Zubair, M., Alzaatreh, A., Cordeiro, G.M., Tahir, M.H. and Mansoor, M. On Generalized Classes of Exponential Distribution using T-X Family Framework. Filomat. $32: 4.2018,1259$

[7] Famoye, F., Akarawak, E. and Ekum, M. Weibull-Normal Distribution and its Applications, Journal of Statistical Theory and Applications. 2018, 17(4), 719 -727.

[8] Ogunsanya A. S., Sanni O.O. M. and Yahya W. B. Exploring Some Properties of Odd Lomax-Exponential Distribution. Annals of Statistical Theory and Applications, 2019,1:21-30

[9] Afify A.Z., Nofal, Z.M., Ebraheim, A.N. Exponentiated Transmuted Generalized Rayleigh Distribution: A New Four Parameter Rayleigh Distribution. Pak.j.stat.oper.res. 2015; 11(1) 115-134.

[10] Almarashi A M, Badr M M., Elgarhy M, Jamal F and Chesneau C. Statistical Inference of the Half-Logistic Inverse Rayleigh Distribution. Entropy 2020, 22, 449.

[11] Mohammed, H.F.; Yahia, N. On type II Topp-Leone inverse Rayleigh distribution. Appl. Math. Sci., 2019, 13, 607 - 615

[12] Ahmad, A.; Ahmad, S.P.; Ahmed, A. Transmuted inverse Rayleigh distribution: A generalization of the inverse Rayleigh distribution. Math. Theory Model. 2014, 4, 90- 98.

[13] Elgarhy, M. and Alrajhi, S. The odd Frechet inverse Rayleigh distribution: Statistical properties and applications. J. Nonlinear Sci. Appl., 2019, 12, 291- 299

[14] Bourguignon, M., Silva, R.B. and Cordeiro, G.M. The Weibull-G family of probability distributions, J. Data Sci. 2014, 1253- 1268. doi.org/10.1080/00401706.1974.104891 50

[15] Galton, F. Enquiries into Human Faculty and its Development. Macmillan and Company, London. 1983.

[16] Moors, J.J. A quantile alternative for kurtosis, The Statistician, 1988, 37, 25-32. 
[17] Gradshteyn, I.S, Ryzhik, I.M. Tables of integrals, series and products, 7th edn. Elsevier, Inc., London. 2007

[18] Ekum, M. I., Adamu, M. O. and Akarawak, E. E. T-Dagum: A Way of Generalizing Dagum Distribution Using Lomax Quantile Function. Journal of Probability and Statistics. Vol. 2020, ID 1641207. https://doi.org/10.1155/2020/1641207.

[19] A. S Ogunsanya, E. E. E. Akarawak, W. B Yahya. A New Ouantile Estimation Method of Weibull-Ravleigh Distribution- Asian Journal of Probability and Statistics, 2020. 9(1), 28-37

[20] Sen, Pranab K., Keating, J. P.; Mason, R. L.. Pitman's measure of closeness: A comparison of statistical estimators. Philadelphia: SIAM. 1993

[21] Choudhuri, N; Ghosal, S; Roy, A. Bayesian Methods for Function Estimation. Handbook of Statistics. Bayesian Thinking. 2005, 25, 373-414. CiteSeerX 10.1.1.324.3052. doi:10.1016/s0169-7161(05)25013-7. 9780444515391.

[22] Kurtz, David M.; Esfahani, Mohammad S.; Scherer, Florian; Soo, Joanne; Jin, Michael C.; Liu, Chih Long; Newman, Aaron M.; Dührsen, Ulrich; Hüttmann, Andreas. "Dynamic Risk Profiling Using Serial Tumor Biomarkers for Personalized Outcome Prediction". Cell. 2019. 178 (3): 699_ 713.e19. doi:10.1016/j.cell.2019.06.011. ISSN 1097-4172. PMID 31280963

[23] Adegoke, T. M., Nasiri, P., Yahya, W. B., Adegoke, G. K., Afolayan, R. B., and Yahaya, A. M. Bayesian Estimation of Kumaraswamy Distribution under Different Loss Functions. Annals of Statistical Theory and Applications, 2019, 2, 90 102.

[24] Preda, V., Panaitescu, E., and Constantinescu, A. Bayes estimators of Modified-Weibull distribution parameters using Lindley's approximation. WSEAS Transactions on Mathematics, 2010, 9(7), 539-549. Varian, H.R. A
Bayesian Approach to Real Estate Assessment, North Holland, Amsterdam, 1975, 195-208.

[25] Zellner, A. Bayesian estimation and prediction using asymmetric loss function, Journal of American Statistical Association, 1986, 81, 446-451.

[26] Soliman, A. A. Reliability estimation in a generalized life-model with application to the Burr-XII. IEEE Trans. Reliabil., 2002, 51, 337-343.

[27] Singh, U., Gupta, P. K., and Upadhyay, S. K. Estimation of parameters for exponentiated Weibull family under Type-II censoring scheme. Computat. Statist. Data Anal, 2005; 48(3), 509-523.

[28] Ahmadi, J., Doostparast, M., and Parsian, A. Estimation and prediction in a two-parameter exponential distribution based on k-record values under LINEX loss function. Commun. Statist. Theor. Meth., 2005, 34, 795-805.

[29] Basu, A. P., and Ebrahimi, N. Bayesian approach to life testing and reliability estimation using asymmetric loss function. J. Statist. Plan. Inference, 1991, 29, 21-31.

[30] Calabria, R., and Pulcini, G. An engineering approach to Bayes estimation for the Weibull distribution. Microelectron Reliability1, 1994, 789-802.

[31] Dey, D. K., Ghosh, M., and Srinivasan, C. Simultaneous Estimation of Parameters under Entropy Loss. J. Statistical Planning and Inference, 1987, 15, 347-363.

[32] Dey, D. K., and Pei-San, L. L. (1992). On comparison of estimators in a generalized life model. Microelectron. Reliab., 32, 207-221.

[33] Badar, M. G. and Priest, A. M. Statistical aspects of fiber and bundle strength in hybrid composites. In T. Hayashi, K. Kawata, and S. Umekawa (eds.), Progress in Science and Engineering Composites, 1982. 1129-1136. 